\title{
Two microRNA regulatory circuits set start and end times for dendritic arborization of a nociceptive neuron
}

Nobuko Suzuki ${ }^{1}$, Hui Chiu ${ }^{1 * \dagger}$, Yan Zou ${ }^{1,2 \dagger}$, Meiyu Shao ${ }^{1}$, Wei Zou ${ }^{3}$, Kang Shen ${ }^{4}$, and Chieh Chang ${ }^{1,5}$

${ }^{1}$ Department of Biological Sciences, University of Illinois at Chicago, Chicago, IL 60607, USA

${ }^{2}$ School of Life Science and Technology, ShanghaiTech University, Shanghai 201210, China

${ }^{3}$ The Fourth Affiliated Hospital, Zhejiang University School of Medicine, Yiwu 322000, China

${ }^{4}$ Howard Hughes Medical Institute, Department of Biology, Stanford University, Stanford, CA 94305, USA

${ }^{5}$ Correspondence: chieh.chang1@gmail.com

*Present address: Division of Biology and Biological Engineering, Howard Hughes

Medical Institute, TianQiao and Chrissy Chen Institute for Neuroscience, California

Institute of Technology, Pasadena, CA 91125, USA

${ }^{\dagger}$ These authors contributed equally 


\section{Summary}

Choreographic dendritic arborization takes place within a defined time frame, but the timing mechanism is currently not known. Here, we report that a precisely timed lin-4-lin14 regulatory circuit triggers an initial dendritic growth activity whereas a precisely timed let-7-lin-41 regulatory circuit signals a subsequent developmental decline in dendritic growth ability, hence restricting dendritic arborization within a defined time frame. Lossof-function mutations in the lin-4 microRNA gene cause limited dendritic outgrowth whereas loss-of-function mutations in its direct target, the lin-14 transcription factor gene, cause precocious and excessive outgrowth. In contrast, loss-of-function mutations in the let-7 microRNA gene prevent a developmental decline in dendritic growth ability whereas loss-of-function mutations in its direct target, the lin-41 tripartite motif protein gene, cause further decline. lin-4 and let-7 regulatory circuits are expressed at the right place and the right time to set start and end times for PVD dendritic arborization. Replacing the endogenous lin-4 promoter at the lin-4 locus with a late-onset let-7 promoter delays PVD dendrite arborization whereas replacing the endogenous let-7 promoter at the let-7 locus with an early-onset lin-4 promoter causes precocious decline in dendritic growth ability in PVD neurons. We further find that lin-28 acts upstream of let-7 in regulating developmental decline in dendritic growth ability. Our results indicate that the lin-4-lin-14 and the lin-28-let-7-lin-41 regulatory circuits control the timing of PVD dendrite arborization through antagonistic regulation of the DMA-1 receptor level on PVD dendrites. 
bioRxiv preprint doi: https://doi.org/10.1101/2021.08.31.458402; this version posted September 1, 2021. The copyright holder for this preprint (which was not certified by peer review) is the author/funder, who has granted bioRxiv a license to display the preprint in perpetuity. It is made available under aCC-BY-NC 4.0 International license.

Keywords: the lin-4-lin-14 pathway, the lin-28-let-7-lin-41 pathway, developmental timing genes, heterochronic genes, neuronal timers, temporal regulation, dendrite arborization, PVD neurons, Caenorhabditis elegans 


\section{Introduction}

Studies on temporal control of cell cycle progression, circadian rhythm, and segmentation have frequently converged on the concept of biological oscillators ${ }^{1-14}$. Biological oscillators are systems of molecules with various levels of expression and activity that act as molecular "clocks" that determine biological rhythms resilient to changes in external environments. Understanding these molecular oscillators gives a glimpse into the mechanism of timing control for the cyclical nature of these processes. However, timing control for non-cyclical biological processes is less understood. Most non-cyclical events in non-neuronal cells are transient, which makes it difficult to study their temporal regulation. In contrast, differentiation of neurons occurs in a longer timescale because of their complex structures, thereby providing us with an opportunity to study intrinsic timing mechanisms.

Although abundant knowledge has been learnt in past decades on temporal control of cell fate specification of neurons ${ }^{15-17}$, less is known for timing of their wiring to give rise to complex neuronal circuits and for timing of their plasticity. In the nervous system, establishment of neuronal connectivity during development and decline in neuronal plasticity during aging are controlled with temporal precision, but the timing mechanisms are largely unknown. The heterochronic pathways are important temporal regulators of animal development and involve a number of microRNA-regulated post-transcriptional genetic circuits, including important interactions between the lin-4 microRNA and its direct target, the lin-14 transcription factor gene $\mathrm{e}^{18,19}$ and between the let- 7 microRNA and its direct target, the $l i n-41$ tripartite motif (TRIM) protein gene ${ }^{20,21}$. Since the discovery of 
the lin-4-lin-14 and the let-7-lin-41 regulatory circuits broadly expressed in the nervous system $^{22,23}$, evidences indicating a widespread role of the lin-4-lin-14 and the let-7-lin-41 regulatory circuits in timing neuronal assembly and plasticity start to accumulate. Distinct microRNA regulatory circuits were recently shown to control orderly neuronal connectivity and regeneration potential decline ${ }^{22-25}$. The lin-4 microRNA and the LIN-14 transcription factor regulate transition of sequential events in AVM neuronal connectivity. Up-regulation of lin-4 and down-regulation of lin-14 signal the end of netrin-mediated axon pathfinding to allow synapse formation in AVM neurons ${ }^{22,24}$. Similar functions of lin4 and lin-14 in temporal regulation of axon pathfinding have been reported in HSN and PLM neurons ${ }^{26,27}$. The lin-4-lin-14 regulatory circuit also temporally controls synaptic rewiring of postmitotic DD motor neurons ${ }^{28,29}$. In postmitotic PVT interneurons, lin-14 temporally regulates onset of zig gene expression, which is required for maintenance of ventral nerve cord structure ${ }^{30}$. The let-7 microRNA and the LIN-41 tripartite motif protein control timing of a post-differentiation event in AVM neurons ${ }^{23,25}$. The progressive increase of let-7 and the progressive decrease of lin-41 contribute to a normal developmental decline in AVM axon regeneration. lin-4 and let-7 microRNAs also regulate the establishment of axodendritic polarity in the DA9 motor neuron early in development and its maintenance at the adult stage, respectively ${ }^{31}$. Despite advances in understanding timing of neuronal connectivity and regeneration decline, intrinsic timing mechanisms that control choreographic dendritic arborization, an integral part of establishing a functional neural circuit, are still elusive. 
Dendritic arborization of the PVD nociceptive neurons takes place within a defined time period starting at the L2 stage and ending at the young adult stage during C. elegans development. The PVD dendritic arbor is established by a complex but well-ordered array of non-overlapping sister dendrites. The creation of this structure involves a stereotypical series of branching decisions, which makes PVD neurons an ideal model system to study timing mechanisms of dendritic arborization. A $1^{\circ}$ dendrite extends from the PVD cell body along the $\mathrm{A} / \mathrm{P}$ axis at the location of the lateral nerve fascicle. Orthogonal arrays of $2^{\circ}, 3^{\circ}$, and $4^{\circ}$ dendritic branches envelop the animal in a manner that alternates between the $\mathrm{D} / \mathrm{V}$ and the $\mathrm{A} / \mathrm{P}$ axes to produce an elaborate network of sensory processes. Growth of these dendrite branches depends on the DMA-1 dendrite receptor in PVD neurons ${ }^{32}$. What timing mechanisms initiate and terminate the arborization of this complex dendritic network? To answer these questions, we search for molecules in PVD neurons that may provide intrinsic temporal controls for dendritic arborization. We identify two timing regulatory circuits that restrain the growth of PVD dendrites within a set time frame. The initial dendritic outgrowth in PVD neurons is actuated by a precisely timed lin-4-lin-14 regulatory circuit, which positively regulates the DMA-1 dendrite receptor level. The dendritic growth is subsequently slowed down by a precisely timed lin-28-let-7-lin-41 regulatory circuit, which negatively regulates the DMA-1 dendrite receptor level, as dendritic arborization comes to an end. 


\section{Results}

\section{lin-4 and let-7 are temporally expressed in PVD neurons during dendrite arborization}

To identify timing mechanisms that restrict PVD dendritic arborization within a defined time period, we sought to identify molecules that are expressed in PVD neurons and whose expression coincides with start and end times of PVD dendritic arborization. We discovered lin-4 and let-7 microRNAs to be excellent candidates that fit both criteria. To understand the temporal expression of lin-4 and let-7 genes in PVD neurons, we developed a $1.9-\mathrm{kb}$ lin-4 promoter reporter and a $2.9-\mathrm{kb}$ let-7 promoter reporter that were stably expressed in PVD (Figure 1; Table S2). The expression levels of these two promoter reporters in the whole animal at different stages correlated strongly with the whole animal stem-loop reverse transcription polymerase chain reaction (RT-PCR) quantification of lin4 and let-7 microRNAs, indicating that these two reporters are reliable ${ }^{22,23}$. Using these reporters, we determined the timing of lin-4 and let-7 expression in PVD during dendritic arborization (Figures $1 \mathrm{~J}$ and $1 \mathrm{~K}$ ). The lin-4 reporter was expressed highly in PVD at the late L2-early L3 stage, when the secondary dendrites start elaborating (Figures 1G, 1H, and $1 \mathrm{~J})$. In contrast, the let-7 reporter was expressed at relatively low levels in PVD at the L3 stage, but was significantly elevated from the L4 stage onward, when growth of the terminal quaternary dendrites comes to an end (Figures 1G, 1I, and 1K). These results indicate that lin-4 and let-7 are expressed at the right place and the right time to initiate and terminate PVD dendritic arborization, respectively.

\section{The lin-4-lin-14 regulatory circuit initiates dendritic outgrowth in PVD neurons}


We previously reported that the lin-4 microRNA represses the expression of the LIN-14 transcription factor to inhibit AVM axon attraction ${ }^{22,24}$. Further analysis of lin-4 and lin-14 reporters revealed overlapping expression of two genes in many other neurons, including PVD, at the early L3 stage (Figures $1 \mathrm{~A}-1 \mathrm{C}$ and $1 \mathrm{H}$ ), when PVD neurons are sending out the secondary dendrites. We showed that lin-4(e912) loss-of-function (lf) and lin-14(n355) gain-of-function $(g f)$ mutants displayed a similar phenotype of limited dendritic outgrowth in PVD neurons (Figures 2A-2C and 2E), suggesting that the lin-4 microRNA may inhibit the expression of the LIN-14 transcription factor to initiate dendrite outgrowth. Consistent with this interpretation, reduced lin-14 activity caused opposite effects. In wild-type animals at the mid L3 stage, PVD dendrites can only grow up to the tertiary branch (Figure 2F). However, in lin-14(n179) reduction-of-function (rf) mutants at the same developmental stage, the mid L3 stage, PVD dendrites can grow up to the quaternary branch and complete the menorah organization (Figure $2 \mathrm{~F}$ ), suggesting precocious dendrite outgrowth. In addition, the number of overlapped tertiary dendrites caused by excessive tertiary dendrite growth was significantly higher in lin-14(n179rf) mutants than in wildtype animals at the young adult stage (Figures $2 \mathrm{~A}, 2 \mathrm{D}, 2 \mathrm{G}$, and $\mathrm{S} 1$ ). Thus, a reduction of function in the lin-14 gene causes precocious and excessive dendritic outgrowth. Precocious PVD dendritic outgrowth in lin-14(n179rf) mutants was not caused by precocious PVD cell fate specification since PVD cells were not specified prematurely in lin-14(n179rf) mutants (data not shown). The lin-14(n179rf) mutant phenotype of excessive PVD tertiary dendrite growth can be rescued by re-expressing the lin-14 gene in the PVD neuron, suggesting that lin-14 acts cell-autonomously in PVD (Figure 2G). To further strengthen the statement that the lin-4 microRNA targets the lin-14 transcription 
factor to initiate dendritic outgrowth in PVD neurons, we tested whether lin-14(n179rf) mutations suppress the lin-4(e912lf) mutant phenotype of limited dendritic outgrowth. We found that this is indeed the case (Figure 2F). lin-4(lf); lin-14(rf) double mutants displayed precocious dendritic outgrowth phenotypes of the lin-14(n179rf) single mutant rather than limited dendritic outgrowth phenotypes of the lin-4(lf) single mutant (Figure 2F). Taken together, our results indicate that the lin-4-lin-14 regulatory circuit initiates dendritic outgrowth in PVD neurons (Figure 7E).

The let-7-lin-41 regulatory circuit slows down dendrite growth in the final stage of dendrite arborization

We previously reported that the let-7 microRNA represses the expression of the LIN-41 tripartite motif protein to inhibit AVM axon regeneration in older neurons ${ }^{23,25}$. Here, we further studied the expression of let-7 and lin-41 reporters and found overlapping expression of two genes in many other neurons, including PVD, at the L4 stage (Figures 1D-1F and 1I), when growth of the terminal quaternary dendrites in PVD comes to an end. We performed laser dendritomy on the primary dendrite in PVD neurons at different developmental stages and found that PVD dendritic growth ability is significantly lower at the adult stage than at the L3 stage (Figures 3A, 3B, and 3E), suggesting that PVD neurons undergo a developmental decline in dendritic growth ability. Our expression analysis showed that the let-7 expression in PVD was at relatively low levels at the L3 stage, but was significantly elevated from the L4 stage onward, which implicates its contribution to the developmental decline in PVD dendritic growth ability (Figure 1K). Indeed, the dendritic growth ability in adult let-7 mutants was indistinguishable from that seen in wild- 
type animals at an earlier developmental stage, the L3 stage (Figures 3A-3E), suggesting that let-7 mutations may retard a normal developmental decline in dendritic growth ability. In the young adult stage, while let-7(n2853rf) mutations significantly enhanced, lin41(n2914lf) mutations significantly reduced dendritic growth ability in PVD neurons (Figure 3F). The lin-41(n2914lf) mutant phenotype in PVD dendrites can be rescued by reexpressing the lin-41 gene in the PVD neuron, suggesting that lin-41 acts cellautonomously in PVD (data not shown). In addition, lin-41 mutations suppressed the let7(n2853rf) mutant phenotype of enhancing dendrite growth ability (Figure 3F). Together, these results indicate that the let-7-lin-41 regulatory circuit slows down dendrite growth in the final stage of dendrite arborization (Figure 7E).

\section{lin-28 inhibits the let-7-lin-41 circuit to regulate the timing of dendrite arborization}

Previous studies have shown that the LIN-28 RNA-binding protein blocks maturation of the let-7 microRNA in both invertebrates and vertebrates ${ }^{33-36}$. We found that a lin-28::GFP fosmid-based reporter is expressed in PVD neurons, which contains the lin-28 upstream cis-regulatory, exonic, intronic, and downstream cis-regulatory sequences (Figure 3G). To determine whether lin-28 acts upstream of let-7 to regulate the timing of PVD dendrite arborization, we first compared the endogenous LIN-41 protein level in PVD neurons between wild type and lin-28(n719lf) mutants. The $\mathrm{mNG}$ reporter gene was knocked in the endogenous $\operatorname{lin}-41$ locus to generate a $m N G::$ lin-41 fusion gene using the CRISPR/Cas9 technology. We found that lin-28(n719lf) mutations significantly reduced LIN-41 protein levels in PVD neurons compared to wild type at the early L3 stage (Figures 3H and 3I), suggesting that lin-28 inhibits the let-7-lin-41 circuit in PVD neurons (Figure 7E). We 
performed laser dendritomy on PVD primary dendrites in lin-28(n719lf) mutants and observed significantly reduced dendrite growth ability in lin-28 (n719lf) mutants compared to wild-type animals (Figure 3J), a phenotype that is opposite to the let-7(n2853rf) mutant phenotype of enhanced dendrite growth ability (Figure 3F). Furthermore, lin-41 overexpression in PVD neurons suppressed the lin-28(n719lf) mutant phenotype of reduced dendrite growth ability (Figure 3J). Together, these results support that lin-28 inhibits the let-7-lin-41 circuit to regulate the timing of PVD dendrite arborization.

Recent studies in sexually dimorphic nervous system differentiation and male tail tip morphogenesis revealed that the lep-5 lncRNA brings together LIN-28 and the LEP-2 Makorin to promote ubiquitination and degradation of LIN-28 $8^{37,38}$. To determine whether lep-5 plays a role in regulating PVD dendrite growth ability, we first analyzed the endogenous LIN-41 protein level in PVD neurons in wild-type animals versus lep5(ny28lf) mutants. We found there is no difference of LIN-41 protein levels in PVD neurons between wild type and lep-5(ny28lf) mutants (Figures S2A and S2B). In addition, lep-5(ny28lf) mutants displayed similar dendrite growth ability to wild-type animals (Figure S2C). Thus, lep-5 is unlikely to regulate the timing of PVD dendrite arborization by modulating the lin-28-let-7-lin-41 circuit.

\section{The lin-4 to let-7 promoter replacement delays dendrite arborization}

To further support our statement that lin-4 and let-7 microRNAs set start and end times for PVD dendrite arborization, we manipulated the timing of their expression by swapping their promoters with each other. We found that delayed expression of lin-4 (lin-4 
microRNA expressed from the let-7 promoter from the L3 stage onward) postponed dendrite arborization in PVD neurons (Figure S3). The transgene that expressed the lin-4 microRNA from the let-7 promoter (Plet-7::lin-4) led to retarded growth in quaternary dendrites (the final order of PVD dendrites) in a lin-4 loss-of-function mutant (Figures S3A and S3B). However, the growth of quaternary dendrites in these transgenic animals (lin4(e912lf); Ex[Plet-7::lin-4]) was able to catch up later in the adult stage (Figure S3C). To further strengthen this conclusion, we utilized the CRISPR/Cas9 technology to replace the endogenous lin-4 promoter at the lin-4 locus with a late-onset let-7 promoter (Figure 4A'). The repair templates that have been developed recently were used to facilitate the identification of the CRISPR recombinants ${ }^{39,40}$ lin-4(xr70) and lin-4(xr71) in which the endogenous lin-4 promoter has been replaced by the let-7 promoter. We used stem-loop RT-PCR to globally survey temporal expression of mature lin-4 microRNA during animal development. Expression of the lin-4 microRNA in the lin-4 to let-7 promoter replacement CRISPR allele was indeed delayed compared to its expression in wild-type animals (Figure 4A). In these CRISPR lines, lin-4(xr70) and lin-4(xr71), we observed retarded growth of the quaternary dendrites at the early L4 stage (Figure 4D compared to 4B of WT; Figure 4F), which was able to catch up later in the adult stage (Figure 4E compared to 4C of WT; Figures 4G-4I). These results demonstrate that, by manipulating the timing of lin-4 expression through the lin-4 to let-7 promoter replacement, we can delay dendrite arborization.

The let-7 to lin-4 promoter replacement precociously inhibits dendrite growth ability 
Conversely, premature expression of let-7 (let-7 microRNA expressed from the lin-4 promoter from the L1 stage onward) precociously inhibited dendritic growth ability in PVD neurons (Figures 5A and 5B). We utilized the CRISPR/Cas9 technology to generate the let-7(xr67) CRISPR line in which the endogenous let-7 promoter at the let-7 locus has been replaced with an early-onset lin-4 promoter (Figure 5A'). Stem-loop RT-PCR analysis showed that expression of mature let-7 microRNA in the let-7 to lin-4 promoter replacement CRISPR line was indeed precocious compared to its expression in wild-type animals (Figure 5A). In the let-7(xr67) CRISPR line, PVD dendrite growth ability at the L3 stage was significantly lower than that in wild-type animals at the L3 stage and similar to that in wild-type animals at the young adult stage (Figure 5B). PVD dendrite growth ability in the let-7(xr67) line was further reduced at the young adult stage (Figure 5B). Interestingly, PVD dendrites, both proximal and distal segments to the injured site, in let7(xr67) animals degenerated 24 hours following laser dendritomy at the D1 A stage (one day into the adult stage). Because of this reason, we were unable to determine the dendrite growth ability at the D1 A stage (Figure 5B). Thus, by manipulating the timing of let-7 expression through the let-7 to lin-4 promoter replacement, we can precociously inhibit dendrite growth ability. Taken together, these findings support a model in which the lin-4lin-14 regulatory circuit sets the start time whereas the let-7-lin-41 regulatory circuit sets the end time for PVD dendrite arborization (Figure 7E).

\section{lin-14 and lin-41 antagonistically regulate the DMA-1 receptor level on PVD}

\section{dendrites}


Two transmembrane ligands, SAX-7 and MNR-1, work together with LECT-2, a secreted ligand, to instruct dendrite arborization in PVD neurons through direct interactions with the dendrite receptor DMA-1 ${ }^{32,41-44}$. SAX-7, MNR-1, LECT-2 and DMA-1 form a multiprotein receptor-ligand signaling complex that directs the growth of stereotyped dendritic branches. One way to control dendrite growth ability during PVD dendrite arborization is through regulation of responsiveness of dendrites to growth signals, which can be accomplished by adjusting the abundance of the DMA-1 receptor on dendrites ${ }^{45}$. To determine whether the lin-4-lin-14 and the lin-28-let-7-lin-41 regulatory circuits control PVD dendrite arborization through the regulation of the DMA-1 receptor on PVD dendrites, we analyzed the level of DMA-1 proteins on specific PVD dendrite branches in lin-14(n179rf) and lin-41(n2914lf) mutants versus wild-type animals. Using the CRISPR/Cas9 technology to knock in the YFP reporter gene to the 3 ' end of the endogenous $d m a-1$ locus to generate a $d m a-1:: y f p$ fusion gene $^{46}$, we showed that the endogenous level of DMA-1 proteins based on the fluorescent intensity of YFP was significantly increased on the tertiary dendrites in lin-14(n179rf) mutants (Figures 6A-6C), consistent with higher dendrite growth ability, which is opposite to lower dendrite growth ability displayed by lin-4(e912lf) mutants (Figure 6D). In contrast, the endogenous level of DMA-1 proteins was significantly reduced on the quaternary dendrites in lin-41(n2914lf) mutants (Figures 7A-7C), consistent with lower dendrite growth ability. This C-terminal tagging seems to partially affect the function of DMA-1 receptors as the number of tertiary and quaternary dendrites is reduced compared to wild-type animals. To further examine the relationship between the lin-4-lin-14 regulatory circuit and $d m a-1$, we analyzed the dendrite growth ability in $d m a-1(x r 50 l f)$; lin-14(n179rf) double mutants. The dma- 
$1(x r 50 l f)$ mutation, which significantly reduced tertiary dendrite outgrowth, suppressed the excessive tertiary dendrite outgrowth caused by the lin-14(n179rf) mutation (Figure 6E). These results suggest that the lin-4-lin-14 regulatory circuit promotes dendrite outgrowth through up-regulation of the DMA-1 receptor level on dendrites (Figure 7E). To further investigate the relationship between the lin-28-let-7-lin-41 regulatory circuit and $d m a-1$, we performed laser dendritomy on PVD neurons in lin-41(ma104rf) mutants with or without $d m a-1$ overexpression in PVD neurons. The $d m a-1$ (xr50lf) mutation significantly reduced dendrite growth ability (Figure 7D). In contrast, $d m a-1$ overexpression in PVD neurons, which significantly enhanced dendrite growth ability, suppressed the reduced dendrite growth ability caused by the lin-41(ma104rf) mutation (Figure 7D). Thus, the lin28-let-7-lin-41 regulatory circuit inhibits dendrite growth ability through down-regulation of the DMA-1 receptor level on dendrites (Figure 7E). lin-41 has been reported to negatively regulate the lin-29 zinc finger transcription factor in certain cellular contexts in C. elegans ${ }^{15,21,23,47}$. Although lin-29 is expressed in PVD neurons strongly from the L4 stage onward (Figures S4A and S4B), the lin-29(n333lf) mutation did not affect dendrite growth ability (Figure S4C). Thus, lin-29 is unlikely to act between lin-41 and $d m a-1$ to regulate the timing of PVD dendrite arborization. Together, these results support a model in which the lin-4-lin-14 and the lin-28-let-7-lin-41 regulatory circuits control the timing of PVD dendrite arborization through antagonistic regulation of the DMA-1 receptor level on PVD dendrites (Figure 7E). 


\section{Discussion}

PVD neurons lose dendrite growth ability as they age, but it is not known why. One theory is that the developmental decline in dendrite growth ability provides a switch in intrinsic states from long-range exploratory growth to short-range dendritic targeting. In this report, we show that two microRNA regulatory circuits are used in timing dendritic arborization in postmitotic PVD neurons, restricting dendrite growth within a defined time frame. The precisely timed lin-4-lin-14 regulatory circuit sets off initial dendrite outgrowth until the L4 stage, at which time point the precisely timed lin-28-let-7-lin-41 regulatory circuit decelerates dendrite growth as terminal dendrite branches are reaching final targets (Figure 7E). These two regulatory circuits control the timing of PVD dendrite arborization through opposed regulation of the DMA-1 receptor level on PVD dendrites (Figure 7E).

The neuronal timers that restrain dendrite arborization within a specific time window during neural circuit formation are poorly understood and remain a mystery. In this study, we uncover two microRNA regulatory circuits that temporally control the choreography of dendrite arborization in PVD neurons. Our study illustrates how neuronal timers regulate the intrinsic potential of dendrite growth. From this point forward, our goal is to describe the required timing mechanisms in dendritic arborization at a resolution that ultimately will allow us to reconstitute the process. Further dissection of genetic networks that regulate the timing of lin-4 and let-7 expression, and identification of downstream targets of the LIN-14 transcription factor and the LIN-41 tripartite motif protein that regulate the DMA1 receptor level will be indispensable to achieving this goal. 
Here, we show that the lin-4-lin-14 regulatory circuit initiates PVD dendrite arborization through up-regulation of the DMA-1 receptor level. However, the $d m a-1$ gene is unlikely a direct target of the LIN-14 transcription factor as the lin-14 mutation not only enhanced the expression of the endogenous $d m a-1$ gene from its own promoter (Figure 6), but also enhanced the expression of the $d m a-1$ transgene from a heterologous ser-2 promoter (data not shown). We report that the let-7-lin-41 regulatory circuit slows down PVD dendrite arborization as it comes to an end through down-regulation of the DMA-1 receptor level. The regulation of $d m a-1$ expression by lin-41 appears to be dependent on regulatory sequences outside of the $d m a-1$ coding region since the expression of the endogenous $d m a-$ 1 gene from the $d m a-1$ locus (Figure 7), but not the expression of the $d m a-1$ transgene from a heterologous ser-2 promoter and a control unc-54 3' UTR is reduced in lin-41(lf) mutants (data not shown). It remains to be seen whether lin-41 functions as RNA-binding proteins to post-transcriptionally regulate the $d m a-1$ expression that is dependent on either 5 ' UTR or 3' UTR of the $d m a-1$ gene ${ }^{47}$. Although lin-29, a known direct target of LIN-41 in certain cellular contexts ${ }^{15,21,23,47}$, is expressed in PVD neurons (Figures S4A and S4B), no enhancement in dendrite growth ability by the lin-29(n333lf) mutation was observed (Figure S4C), which suggests that lin-29 is unlikely to play a role in regulating the timing of PVD dendrite arborization. Thus, further studies are required to understand the mechanisms by which LIN-14 and LIN-41 regulate the DMA-1 receptor level on PVD dendrites.

In the let-7(xr67) CRISPR line, the let-7 microRNA expressed from the lin-4 promoter does not block PVD dendrite growth in early development. This could be due to many 
different reasons. For example, the Plin-4::let-7 genomic configuration may not be able to support expression and processing of the primary let-7 microRNA as efficiently as the Plin$4: \because$ lin-4 genomic configuration supports expression and processing of the primary lin-4 microRNA. Alternatively, between the lin-4-lin-14 and the let-7-lin-41 regulatory circuits, the lin-4-lin-14 regulatory circuit could impact the DMA-1 receptor level to a larger extent than the let-7-lin-41 regulatory circuit.

It remains to be seen whether the timing mechanisms of dendrite arborization by lin-4 and let-7 microRNA regulatory circuits can be extended beyond PVD neurons, especially knowing that these two timing microRNAs are expressed broadly in many neurons in $C$. elegans (Figures 1A-1F) 22,23 . Since let-7 and lin-4 microRNAs are evolutionarily conserved, it is possible that these microRNA regulatory circuits control the timing of dendrite arborization in other organisms as well. This possibility is supported by the findings that let-7 is expressed in the nervous system in various species, including $C$. elegans, Drosophila, mouse, and human ${ }^{20,23,48,49}$ and that $m i R-125$, the lin-4 homolog, functions in the Drosophila and mouse nervous system ${ }^{50,51}$. Future studies in other systems could determine whether temporal control of dendrite arborization by the two microRNA regulatory circuits is also evolutionarily conserved. Although little is known about the timing of dendrite arborization in vertebrates, research has suggested that failed stabilization of dendritic branches in adults could lead to psychiatric and neurodegenerative disorders $^{52}$. Our studies thus provide important mechanistic insights into the timing of dendrite arborization, a critical step in forming functional neuronal circuits. 


\section{Experimental Procedures}

\section{Strains and plasmids}

C. elegans strains were cultured using standard methods ${ }^{53}$. All strains were grown at $20^{\circ} \mathrm{C}$, except for experiments that involved the lin-14(n179ts) allele, which was grown at $25^{\circ} \mathrm{C}$. Standard protocol was used for the plasmid constructions. Strains and plasmids used in this study are listed in Tables S1 and S2.

\section{Transgenic animals}

Germline transformation of $C$. elegans was performed using standard techniques ${ }^{54}$. For example, the Plin-41::GFP transgene was injected at $50 \mathrm{ng} / \mu \mathrm{l}$ along with the coinjection marker Podr-1::RFP at $50 \mathrm{ng} / \mu \mathrm{l}$. Transgenic lines were maintained by following the Podr$1:: R F P$ fluorescence.

\section{CRISPR/Cas9 genome editing}

We generated the lin-4(xr70), lin-4(xr71), let-7(xr67), and let-7(xr68) CRISPR lines using the CRISPR/Cas9 genome editing technology. CRISPR recombinants were identified using a self-excising drug selection cassette as previously described ${ }^{39,40}$. The strategies are illustrated in the diagrams in Figures 4A' and 5A'.

\section{Stem-loop reverse transcription-PCR}

We quantified the mature microRNA level by modifying the microRNA assay developed previously ${ }^{55}$. Equal amounts of the RNA preparation from staged wild type, $\operatorname{lin}-4(x r 71)$, and let-7(xr67) animals were used for RT-PCR amplification of mature microRNAs and of 
pmp-3 transcripts. Reverse transcription reactions contained purified total RNA, $50 \mathrm{nM}$ stem-loop RT primer, 1X RT first strand buffer, $0.25 \mathrm{mM}$ each of dNTPs, $10 \mathrm{mM} \mathrm{MgCl}$, 0.1 M DTT, 200 U SuperScript III reverse transcriptase and 40 U RNase inhibitor. The mixture of RNA template, dNTPs and RT primer was incubated for $5 \mathrm{~min}$ at $65^{\circ} \mathrm{C}$. The mixture was then placed on ice for at least 1 min before adding RT buffer, DTT, $\mathrm{MgCl}_{2}$, SuperScript III and RNase inhibitor. The reaction was incubated for $50 \mathrm{~min}$ at $50^{\circ} \mathrm{C}$ before heat inactivation at $85^{\circ} \mathrm{C}$ for $5 \mathrm{~min}$. PCR was conducted using $0.25 \mu \mathrm{RT}$ products as template in $20 \mu \mathrm{l}$ PCR for 17 cycles.

RT_primer_lin-4 TCAACTGGTGTCGTGGAGTCGGCAATTCAGTTGAGTCACACTT

lin-4_F CGGCGGTCCCTGAGACCTCAA

RT_primer_let-7 CTCAACTGGTGTCGTGGAGTCGGCAATTCAGTTGAGAACTATAC

let-7_F CGGCGGTGAGGTAGTAGGTTGT

Universal reverse primer CTGGTGTCGTGGAGTCGGCAATTC

pmp-3-F TGGCCGGATGATGGTGTCGC

pmp-3-R ACGAACAATGCCAAAGGCCAGC

\section{Laser dendritomy}

Animals were mounted on $2 \%$ agarose pads and anesthetized with $5 \mathrm{mM}$ sodium azide, the lowest possible concentration to keep adult animals immobilized. Laser dendritomy was performed on PVD primary dendrites using either a cavity-dumped Ti:sapphire laser oscillator (Cascade Laser, KMLabs Inc.), which generates laser pulses $100 \mathrm{fs}$ in duration 
and $200 \mathrm{kHz}$ in repetition rate, or a MicroPoint Laser Ablation System (Andor/Oxford Instruments), which generates $337 \mathrm{~nm}$ laser pulses 2-6 ns in duration. The laser pulses were tightly-focused onto targeted primary dendrites using a Plan Apo VC 100x, 1.4 NA oilimmersion objective on a Nikon ECLIPSE Ti microscope. Successful laser dendritomy was confirmed by visualizing the targeted area immediately after surgery. Worms were recovered within 10 minutes of sodium azide treatment and placed on fresh plates with bacterial food.

\section{Quantification of dendrite lengths}

PVD neurons in recovered worms were imaged 24 hours after dendritomy and the dendrite regrowth was quantified. Dendrite lengths were calculated as the actual contour length between the injury site and dendrite termini measured along the cylindrical surface of each worm by tracing dendrites through a 3-dimensional image stack. P values for the length measurements were calculated using a Student's $t$-test.

\section{Fluorescence microscopy}

Animals were mounted on $2 \%$ agarose pads and anesthetized with $20 \mathrm{mM}$ sodium azide. Fluorescence microscopy was performed using either a Plan-Apochromat 60x, 1.4 NA objective on a Zeiss Axio Imager M2 microscope with a Hamamatsu ORCA-Flash4.0 LT+ camera, or a Plan Apo VC 60x, 1.4 NA objective on a Nikon ECLIPSE Ti microscope with a Hamamatsu ORCA-ER camera. The morphology of neuronal cell bodies and dendrites was based on high-magnification Z-stacks. Images of DMA-1-YFP were captured in live 
animals using a Plan-Apochromat 40x, 1.3 NA objective on a Zeiss LSM 880 confocal microscope.

\section{Statistical analysis}

Average data of dendrite number, dendrite length, promoter reporter expression intensity, and relative DMA-1::YFP intensity are presented as means \pm SEM. Data of \% PVD neurons with excessive or limited dendrites are presented as proportions \pm SEP. Statistical analyses were carried out by Student's $t$-tests or two-proportion Z-tests using GraphPad Prism 7.0 or the Primer of Biostatistics software. The statistical test used for each panel is described in the figure legend. Sample sizes for experiments are shown in the respective panels. In all figures, $n$ represents number of animals examined. 


\section{Author Contributions}

N.S. conceived, designed, performed, analyzed experiments, made constructs, and drafted the article. H.C. conceived, designed, performed, analyzed experiments, and drafted the article. Y.Z. conceived, designed, performed, analyzed experiments, and made constructs. M.S. made the promoter replacement CRISPR lines, performed, and analyzed experiments. W.Z. made the dma-1(wy996) [dma-1::YFP] CRISPR line and contributed unpublished essential data and reagents. K.S. conceived experiments, contributed unpublished essential data and reagents, and drafted the article. C.C. conceived, designed, analyzed and interpreted data, and drafted the article.

\section{Acknowledgments}

This work was funded by grants from the March of Dimes Foundation (C.C.), the Whitehall Foundation Research Award (C.C.), the National Science Foundation (IOS-1455758 to C.C.), the National Institute of General Medical Sciences of the National Institutes of Health (R01GM111320 to C.C.), and the Howard Hughes Medical Institute (K.S.). We thank Daniel J. Dickinson and Bob Goldstein for providing reagents and CRISPR protocols, David H.A. Fitch for the lep-5(ny28) mutant allele, Oliver Hobert for the lin29(xe61) reporter strain, Kana Hamada for confocal imaging protocols, Evguenia Ivakhnitskaia for technical assistance and critical reading the manuscript, the Caenorhabditis Genetics Center for strains, and the WormBase for readily accessible information.

\section{Declaration of Interests}


bioRxiv preprint doi: https://doi.org/10.1101/2021.08.31.458402; this version posted September 1, 2021. The copyright holder for this preprint (which was not certified by peer review) is the author/funder, who has granted bioRxiv a license to display the preprint in perpetuity. It is made available under aCC-BY-NC 4.0 International license.

The authors declare no competing interests. 


\section{References}

1. Bargiello, T.A., Jackson, F.R., and Young, M.W. (1984). Restoration of circadian behavioural rhythms by gene transfer in Drosophila. Nature 312, 752-754. $10.1038 / 312752 \mathrm{a} 0$.

2. Cooke, J., and Zeeman, E.C. (1976). A clock and wavefront model for control of the number of repeated structures during animal morphogenesis. J Theor Biol 58, 455-476. 10.1016/s0022-5193(76)80131-2.

3. Hardin, P.E., Hall, J.C., and Rosbash, M. (1990). Feedback of the Drosophila period gene product on circadian cycling of its messenger RNA levels. Nature 343, 536-540. 10.1038/343536a0.

4. Horikawa, K., Ishimatsu, K., Yoshimoto, E., Kondo, S., and Takeda, H. (2006). Noise-resistant and synchronized oscillation of the segmentation clock. Nature 441, 719-723. 10.1038/nature04861.

5. Kondo, T. (2007). A cyanobacterial circadian clock based on the Kai oscillator. Cold Spring Harb Symp Quant Biol 72, 47-55. 10.1101/sqb.2007.72.029.

6. Liu, X., Zwiebel, L.J., Hinton, D., Benzer, S., Hall, J.C., and Rosbash, M. (1992). The period gene encodes a predominantly nuclear protein in adult Drosophila. J Neurosci 12, 2735-2744.

7. Morgan, D.O. (1997). Cyclin-dependent kinases: engines, clocks, and microprocessors. Annu Rev Cell Dev Biol 13, 261-291.

10.1146/annurev.cellbio.13.1.261.

8. Nakajima, M., Imai, K., Ito, H., Nishiwaki, T., Murayama, Y., Iwasaki, H., Oyama, T., and Kondo, T. (2005). Reconstitution of circadian oscillation of cyanobacterial KaiC phosphorylation in vitro. Science 308, 414-415. 10.1126/science. 1108451 .

9. Oates, A.C., Morelli, L.G., and Ares, S. (2012). Patterning embryos with oscillations: structure, function and dynamics of the vertebrate segmentation clock. Development 139, 625-639. 10.1242/dev.063735.

10. Price, J.L., Blau, J., Rothenfluh, A., Abodeely, M., Kloss, B., and Young, M.W. (1998). double-time is a novel Drosophila clock gene that regulates PERIOD protein accumulation. Cell 94, 83-95. 10.1016/s0092-8674(00)81224-6.

11. Reppert, S.M., and Weaver, D.R. (2002). Coordination of circadian timing in mammals. Nature 418, 935-941. 10.1038/nature00965. 
12. Siwicki, K.K., Eastman, C., Petersen, G., Rosbash, M., and Hall, J.C. (1988). Antibodies to the period gene product of Drosophila reveal diverse tissue distribution and rhythmic changes in the visual system. Neuron 1, 141-150. 10.1016/0896-6273(88)90198-5.

13. Vosshall, L.B., Price, J.L., Sehgal, A., Saez, L., and Young, M.W. (1994). Block in nuclear localization of period protein by a second clock mutation, timeless. Science 263, 1606-1609. 10.1126/science.8128247.

14. Zehring, W.A., Wheeler, D.A., Reddy, P., Konopka, R.J., Kyriacou, C.P., Rosbash, M., and Hall, J.C. (1984). P-element transformation with period locus DNA restores rhythmicity to mutant, arrhythmic Drosophila melanogaster. Cell 39, 369-376. 10.1016/0092-8674(84)90015-1.

15. Pereira, L., Aeschimann, F., Wang, C., Lawson, H., Serrano-Saiz, E., Portman, D.S., Grosshans, H., and Hobert, O. (2019). Timing mechanism of sexually dimorphic nervous system differentiation. Elife 8. 10.7554/eLife.42078.

16. Faunes, F., and Larraín, J. (2016). Conservation in the involvement of heterochronic genes and hormones during developmental transitions. Dev Biol 416, 3-17. 10.1016/j.ydbio.2016.06.013.

17. Rougvie, A.E., and Moss, E.G. (2013). Developmental transitions in C. elegans larval stages. Curr Top Dev Biol 105, 153-180. 10.1016/B978-0-12-3969682.00006-3.

18. Lee, R.C., Feinbaum, R.L., and Ambros, V. (1993). The C. elegans heterochronic gene lin-4 encodes small RNAs with antisense complementarity to lin-14. Cell 75, 843-854. 10.1016/0092-8674(93)90529-y.

19. Wightman, B., Ha, I., and Ruvkun, G. (1993). Posttranscriptional regulation of the heterochronic gene lin-14 by lin-4 mediates temporal pattern formation in C. elegans. Cell 75, 855-862. 10.1016/0092-8674(93)90530-4.

20. Pasquinelli, A.E., Reinhart, B.J., Slack, F., Martindale, M.Q., Kuroda, M.I., Maller, B., Hayward, D.C., Ball, E.E., Degnan, B., Muller, P., et al. (2000). Conservation of the sequence and temporal expression of let-7 heterochronic regulatory RNA. Nature 408, 86-89. 10.1038/35040556.

21. Slack, F.J., Basson, M., Liu, Z., Ambros, V., Horvitz, H.R., and Ruvkun, G. (2000). The lin-41 RBCC gene acts in the C. elegans heterochronic pathway between the let-7 regulatory RNA and the LIN-29 transcription factor. Mol Cell 5, 659-669. 10.1016/s1097-2765(00)80245-2. 
22. Zou, Y., Chiu, H., Domenger, D., Chuang, C.F., and Chang, C. (2012). The lin-4 microRNA targets the LIN-14 transcription factor to inhibit netrin-mediated axon attraction. Sci Signal 5, ra43. 10.1126/scisignal.2002437.

23. Zou, Y., Chiu, H., Zinovyeva, A., Ambros, V., Chuang, C.F., and Chang, C. (2013). Developmental decline in neuronal regeneration by the progressive change of two intrinsic timers. Science 340, 372-376. 10.1126/science.1231321.

24. Chiu, H., Alqadah, A., and Chang, C. (2014). The role of microRNAs in regulating neuronal connectivity. Front Cell Neurosci 7, 283. $10.3389 /$ fncel.2013.00283.

25. Chiu, H., and Chang, C. (2013). Rejuvenating nerve cells in adults. Aging (Albany NY) 5, 485-486. 10.18632/aging.100574.

26. Olsson-Carter, K., and Slack, F.J. (2010). A developmental timing switch promotes axon outgrowth independent of known guidance receptors. PLoS Genet 6. 10.1371/journal.pgen.1001054.

27. Xu, Y., and Quinn, C.C. (2016). Transition between synaptic branch formation and synaptogenesis is regulated by the lin-4 microRNA. Dev Biol 420, 60-66. 10.1016/j.ydbio.2016.10.010.

28. Hallam, S.J., and Jin, Y. (1998). lin-14 regulates the timing of synaptic remodelling in Caenorhabditis elegans. Nature 395, 78-82. 10.1038/25757.

29. Howell, K., White, J.G., and Hobert, O. (2015). Spatiotemporal control of a novel synaptic organizer molecule. Nature 523, 83-87. 10.1038/nature14545.

30. Aurelio, O., Boulin, T., and Hobert, O. (2003). Identification of spatial and temporal cues that regulate postembryonic expression of axon maintenance factors in the C. elegans ventral nerve cord. Development 130, 599-610. 10.1242/dev.00277.

31. Armakola, M., and Ruvkun, G. (2019). Regulation of Caenorhabditis elegans neuronal polarity by heterochronic genes. Proc Natl Acad Sci U S A 116, 1232712336. 10.1073/pnas.1820928116.

32. Liu, O.W., and Shen, K. (2012). The transmembrane LRR protein DMA-1 promotes dendrite branching and growth in C. elegans. Nat Neurosci 15, 57-63. 10.1038/nn.2978.

33. Lehrbach, N.J., Armisen, J., Lightfoot, H.L., Murfitt, K.J., Bugaut, A., Balasubramanian, S., and Miska, E.A. (2009). LIN-28 and the poly(U) polymerase PUP-2 regulate let-7 microRNA processing in Caenorhabditis elegans. Nat Struct Mol Biol 16, 1016-1020. 10.1038/nsmb.1675. 
34. Vadla, B., Kemper, K., Alaimo, J., Heine, C., and Moss, E.G. (2012). lin-28 controls the succession of cell fate choices via two distinct activities. PLoS Genet 8, e1002588. 10.1371/journal.pgen.1002588.

35. Van Wynsberghe, P.M., Kai, Z.S., Massirer, K.B., Burton, V.H., Yeo, G.W., and Pasquinelli, A.E. (2011). LIN-28 co-transcriptionally binds primary let-7 to regulate miRNA maturation in Caenorhabditis elegans. Nat Struct Mol Biol 18, 302-308. 10.1038/nsmb.1986.

36. Viswanathan, S.R., Daley, G.Q., and Gregory, R.I. (2008). Selective blockade of microRNA processing by Lin28. Science 320, 97-100. 10.1126/science.1154040.

37. Kiontke, K.C., Herrera, R.A., Vuong, E., Luo, J., Schwarz, E.M., Fitch, D.H.A., and Portman, D.S. (2019). The Long Non-Coding RNA lep-5 Promotes the Juvenile-to-Adult Transition by Destabilizing LIN-28. Dev Cell 49, 542-555 e549. 10.1016/j.devcel.2019.03.003.

38. Lawson, H., Vuong, E., Miller, R.M., Kiontke, K., Fitch, D.H., and Portman, D.S. (2019). The Makorin lep-2 and the lncRNA lep-5 regulate lin-28 to schedule sexual maturation of the C. elegans nervous system. Elife $8.10 .7554 /$ Life. 43660.

39. Dickinson, D.J., Pani, A.M., Heppert, J.K., Higgins, C.D., and Goldstein, B. (2015). Streamlined Genome Engineering with a Self-Excising Drug Selection Cassette. Genetics 200, 1035-1049. 10.1534/genetics.115.178335.

40. Dickinson, D.J., Ward, J.D., Reiner, D.J., and Goldstein, B. (2013). Engineering the Caenorhabditis elegans genome using Cas9-triggered homologous recombination. Nat Methods 10, 1028-1034. 10.1038/nmeth.2641.

41. Díaz-Balzac, C.A., Rahman, M., Lazaro-Pena, M.I., Martin Hernandez, L.A., Salzberg, Y., Aguirre-Chen, C., Kaprielian, Z., and Bulow, H.E. (2016). Muscleand Skin-Derived Cues Jointly Orchestrate Patterning of Somatosensory Dendrites. Curr Biol 26, 2379-2387. 10.1016/j.cub.2016.07.008.

42. Dong, X., Liu, O.W., Howell, A.S., and Shen, K. (2013). An extracellular adhesion molecule complex patterns dendritic branching and morphogenesis. Cell 155, 296-307. 10.1016/j.cell.2013.08.059.

43. Salzberg, Y., Diaz-Balzac, C.A., Ramirez-Suarez, N.J., Attreed, M., Tecle, E., Desbois, M., Kaprielian, Z., and Bulow, H.E. (2013). Skin-derived cues control arborization of sensory dendrites in Caenorhabditis elegans. Cell 155, 308-320. 10.1016/j.cell.2013.08.058. 
44. Zou, W., Shen, A., Dong, X., Tugizova, M., Xiang, Y.K., and Shen, K. (2016). A multi-protein receptor-ligand complex underlies combinatorial dendrite guidance choices in C. elegans. Elife 5. 10.7554/eLife.18345.

45. Dong, X., Chiu, H., Park, Y.J., Zou, W., Zou, Y., Ozkan, E., Chang, C., and Shen, K. (2016). Precise regulation of the guidance receptor DMA-1 by KPC-1/Furin instructs dendritic branching decisions. Elife 5. 10.7554/eLife.11008.

46. Zou, W., Dong, X., Broederdorf, T.R., Shen, A., Kramer, D.A., Shi, R., Liang, X., Miller, D.M., 3rd, Xiang, Y.K., Yasuda, R., et al. (2018). A Dendritic Guidance Receptor Complex Brings Together Distinct Actin Regulators to Drive Efficient F-Actin Assembly and Branching. Dev Cell 45, 362-375 e363. 10.1016/j.devcel.2018.04.008.

47. Aeschimann, F., Kumari, P., Bartake, H., Gaidatzis, D., Xu, L., Ciosk, R., and Grosshans, H. (2017). LIN41 Post-transcriptionally Silences mRNAs by Two Distinct and Position-Dependent Mechanisms. Mol Cell 65, 476-489 e474. 10.1016/j.molcel.2016.12.010.

48. Fairchild, C.L.A., Cheema, S.K., Wong, J., Hino, K., Simo, S., and La Torre, A. (2019). Let-7 regulates cell cycle dynamics in the developing cerebral cortex and retina. Sci Rep 9, 15336. 10.1038/s41598-019-51703-x.

49. Sempere, L.F., Dubrovsky, E.B., Dubrovskaya, V.A., Berger, E.M., and Ambros, V. (2002). The expression of the let-7 small regulatory RNA is controlled by ecdysone during metamorphosis in Drosophila melanogaster. Dev Biol 244, 170179. 10.1006/dbio.2002.0594.

50. Åkerblom, M., Petri, R., Sachdeva, R., Klussendorf, T., Mattsson, B., Gentner, B., and Jakobsson, J. (2014). microRNA-125 distinguishes developmentally generated and adult-born olfactory bulb interneurons. Development 141, 15801588. 10.1242/dev.101659.

51. Wu, Y.C., Chen, C.H., Mercer, A., and Sokol, N.S. (2012). Let-7-complex microRNAs regulate the temporal identity of Drosophila mushroom body neurons via chinmo. Dev Cell 23, 202-209. 10.1016/j.devcel.2012.05.013.

52. Koleske, A.J. (2013). Molecular mechanisms of dendrite stability. Nat Rev Neurosci 14, 536-550. 10.1038/nrn3486.

53. Brenner, S. (1974). The genetics of Caenorhabditis elegans. Genetics 77, 71-94.

54. Mello, C., and Fire, A. (1995). DNA transformation. Methods Cell Biol 48, 451482. 
55. Chen, C., Ridzon, D.A., Broomer, A.J., Zhou, Z., Lee, D.H., Nguyen, J.T., Barbisin, M., Xu, N.L., Mahuvakar, V.R., Andersen, M.R., et al. (2005). Realtime quantification of microRNAs by stem-loop RT-PCR. Nucleic Acids Res 33, e179. 10.1093/nar/gni178. 


\section{Figure legends}

\section{Figure 1. Expression of lin-4/lin-14 and let-7/lin-41 regulatory circuits in PVD}

neurons. (A-C) Overlapping expression of Plin-4::GFP and Plin-14::mCherry reporters in neurons of many regions, such as the head ganglia, ventral nerve cord, tail, and midbody region at the third larval (L3) stage. (D-F) Overlapping expression of Plet-7::GFP and Plin-41::mCherry reporters in many neurons, in the head ganglia, ventral nerve cord, tail, and mid-body region at the L4 stage. PVD neuron expression was also indicated. Scale bar, $20 \mu \mathrm{m}$. (G) The timing and steps of PVD dendrite arborization. Schematic drawings of PVD dendrite arbors as they are seen at five different stages of development, late larval stage 2 (L2) to late L4. All views showing the left-side cells of each PVD pair; anterior is to the left. A single axon (grey color) emerges ventralward from the cell body before traveling anteriorly along the ventral nerve cord. The dendrite processes emerging from the cell body elaborate into highly organized dendrite arbors. (H) Both Plin-4::GFP and Plin14::GFP reporters are expressed in PVD neurons. (I) Detection of both Plet-7::GFP and Plin-41::GFP expression in PVD neurons. Scale bar, $10 \mu \mathrm{m}$. (J) Expression of the Plin$4: \because G F P$ reporter in PVD neurons was assessed at five different stages of development in wild-type animals. (K) Expression of the Plet-7::GFP reporter in PVD neurons was assessed at four different stages of development in wild-type animals. Each line represents data from a single animal followed over time. Bars represent the average expression intensity of either the Plin-4::GFP or the Plet-7::GFP reporter measured at each time point. Error bars, SEM. ***p $<0.001$ by a Student's $t$-test. 
Figure 2. Initiation of dendritic arborization is affected by mutations in the lin-4-lin14 regulatory circuit. (A-D) Representative images showing extent of dendrite arborization in wild type, lin-4(e912lf), lin-14(n355gf), and lin-14(n179rf) mutants. Arrowheads point to contacts between neighboring tertiary dendrites. Scale bar, $20 \mu \mathrm{m}$. (E) Percentages of PVD neurons at the young adult stage with limited dendrite outgrowth in wild type, lin-4(e912lf), and lin-14(n355gf) mutants. Error bars, SEP. ***p $<0.001$, relative to wild type, by a two-proportion Z-test. (F) Percentages of PVD neurons at the mid L3 stage based on the highest order dendrite branch observed in wild type, lin4(e912lf), lin-14(n179rf), and lin-4(e912lf); lin-14(n179rf) mutants. (G) Quantification of the number of tertiary branches per $250 \mu \mathrm{m}$ in the anterior direction from the PVD cell body at the young adult stage in wild type, lin-14(n179rf) mutants, lin-14(n179rf) mutants carrying the Pser-2::lin-14 transgene, animals carrying the Pser-2::lin-14 transgene, and lin-14(n355gf) mutants. Tertiary dendrites were divided into two groups: non-overlapped tertiary is defined as those with normal self-avoidance and overlapped tertiary as those with self-avoidance defects. Error bars, SEM. $* * * p<0.001$ by a Student's $t$-test.

Figure 3. Developmental decline in dendrite growth ability is affected by mutations in the lin-28-let-7-lin-41 regulatory circuit. (A-D) Representative images showing extent of dendrite regrowth 24 hours after dendritomy of the primary dendrite at either the L3 or the young adult stage in wild type $(\mathbf{A}, \mathbf{B})$ and let-7(n2853rf) mutants $(\mathbf{C}, \mathbf{D})$. PVD dendrites were visualized using the $x r I s 37[P F 49 H 12.4: \because G F P]$ marker. Dorsal is up; anterior is to the left. Red arrows indicate lesion sites. Scale bar, $20 \mu \mathrm{m}$. (E) Average PVD dendrite length regrown in wild type and let-7(n2853rf) mutants 24 hours following dendritomy at 
different stages. Asterisks indicate cases in which later stage animals differ from L3-stage animals at $* * * \mathrm{p}<0.001$ by a Student's $t$-test. D1 A indicates one day into the adult stage. Error bars indicate SEM. (F) Average dendrite length regrown in wild type, let-7(n2853rf), lin-41(n2914lf), and lin-41(n2914lf); let-7(n2853rf) mutants. PVD primary dendrites were severed by laser surgery at the young adult stage. Dendrite lengths were measured 24 hours after dendritomy as the actual contour length between the injury site and dendrite termini by tracing dendrites through a 3-dimensional image stack. Asterisks indicate cases in which let-7 or lin-41 mutants differ from wild type at ***p $<0.001$ by a Student's $t$-test. NS, not significant. (G) Representative images of the expression of LIN-28 proteins in PVD neurons in wild type at the early L3 stage. A lin-28::GFP fosmid-based reporter is expressed in PVD neurons. The Pser-2::mCherry reporter was used to label PVD neurons. Scale bar, $5 \mu \mathrm{m}$. (H) Representative images of the expression of endogenous LIN-41 proteins in PVD neurons in wild type and lin-28(n719lf) mutants at the early L3 stage. The Pser-2::mCherry reporter was used to label PVD neurons. Scale bar, $5 \mu \mathrm{m}$. (I)

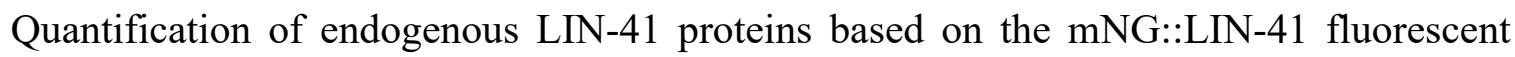
intensity in the PVD cell body in wild type and lin-28(n719lf) mutants. (J) Average dendrite length regrown in wild type, lin-28(n719lf) mutants, and lin-28(n719lf) mutants overexpressing lin-41 in PVD neurons. Error bars, SEM. ***p $<0.001$ by a Student's $t$ test.

Figure 4. Delayed dendrite arborization by the lin-4 to let-7 promoter replacement. Expression of the lin-4 microRNA by a late-onset let-7 promoter postponed growth of the quaternary dendrites. (A) Stem-loop RT-PCR analysis of RNA isolated from populations 
of staged animals revealed late onset expression of the lin-4 microRNA in the $\operatorname{lin}-4(x r 71)$ CRISPR line contrast to early onset expression in wild-type animals. (A') Strategies of promoter replacements by the CRISPR/Cas9 technology. (B-E) Representative images showing extent of quaternary dendrite arborization in wild type $(\mathbf{B}, \mathbf{C})$ and the lin-4 $(x r 71)$ CRISPR line (D, E), in which the endogenous lin-4 promoter has been replaced by the let7 promoter. Images were taken at the early L4 $(\mathbf{B}, \mathbf{D})$ and the young adult $(\mathbf{C}, \mathbf{E})$ stages. Dorsal is up; anterior is to the left. Scale bar, $20 \mu \mathrm{m}$. (F-I) Quantification of the number of quaternary branches per $250 \mu \mathrm{m}$ in the anterior direction from the PVD cell body at the early L4 stage (F) or various time points at the young adult stage (G-I) in wild type, lin4(xr70) CRISPR line, and lin-4(xr71) CRISPR line. Each dot represents data from a single animal. ${ }^{* *} \mathrm{p}<0.001$ by a Student's $t$-test.

Figure 5. Precocious decline in dendrite growth ability by the let-7 to lin-4 promoter replacement. Expression of the let-7 microRNA by an early-onset lin-4 promoter precociously reduced dendrite growth ability in PVD neurons. (A) Stem-loop RT-PCR analysis of RNA isolated from populations of staged animals revealed early onset expression of the let-7 microRNA in the let-7(xr67) CRISPR line contrast to late onset expression in wild-type animals. (A') Strategies of promoter replacements by the CRISPR/Cas9 technology. (B) Average PVD dendrite length regrown in wild type and the let-7(xr67) CRISPR line 24 hours following dendritomy of the primary dendrite at different stages. Asterisks indicate cases in which later stage animals differ from L3-stage animals or a comparison between wild type and let-7(xr67) is significantly different at $* * * p<0.001$ 
by a Student's $t$-test. D1 A indicates one day into the adult stage. ND represents "not determined". Error bars indicate SEM.

Figure 6. lin-14 negatively regulates DMA-1 protein levels on PVD dendrites. (A, B) Representative images showing DMA-1::YFP fusion proteins on PVD dendrites in wild type (A) and lin-14(n179rf) mutants (B). Arrowheads point to tertiary dendrites. Dorsal is up; anterior is to the left. Scale bar, $20 \mu \mathrm{m}$. (C) Average fluorescent intensity of DMA1::YFP fusion proteins on PVD tertiary dendrites in wild type (dma-1(wy996)) versus lin14(n179rf) mutants (dma-1(wy996); lin-14(n179rf)). Error bars, SEM. ***p $<0.001$ by a Student's $t$-test. (D) Quantification of the number of secondary dendrites per $300 \mu \mathrm{m}$ in the anterior direction from the PVD cell body at the young adult stage in wild type and lin4(e912lf) mutants. Each dot represents data from a single animal. (E) Percentages of PVD neurons at the young adult stage with excessive or limited tertiary dendrite outgrowth in lin-14(n179rf), dma-1(xr50lf), and dma-1(xr50lf); lin-14(n179rf) mutants. ***p $<0.001$ by a Student's $t$-test.

Figure 7. lin-41 positively regulates DMA-1 protein levels on PVD dendrites. (A, B) Representative images showing DMA-1::YFP fusion proteins on PVD dendrites in wild type (A) and lin-41(n2914lf) mutants (B). Arrowheads point to quaternary dendrites. (C) Average fluorescent intensity of DMA-1::YFP fusion proteins on PVD quaternary dendrites in wild type (dma-1(wy996)) versus lin-41(n2914lf) mutants (dma-1(wy996) lin41(n2914lf)). (D) Average PVD dendrite length regrown in wild type, lin-41(ma104rf) mutants, dma-1(xr50lf) mutants, animals overexpressing $d m a-1$ in PVD neurons, and lin- 
41(ma104rf) mutants overexpressing $d m a-1$ in PVD neurons 24 hours following dendritomy of the primary dendrite at the young adult stage. Error bars, SEM. ${ }^{*} \mathrm{p}<0.05$ and $* * * p<0.001$ by a Student's $t$-test. (E) Model of sculpting dendritic arbors by two precisely timed microRNA regulatory circuits. Initially, the lin-4 microRNA downregulates the lin-14 transcription factor to trigger dendrite arborization. Later, the let-7 microRNA down-regulates the lin-41 tripartite motif protein to slow down dendrite growth in the final stage of dendrite arborization. 

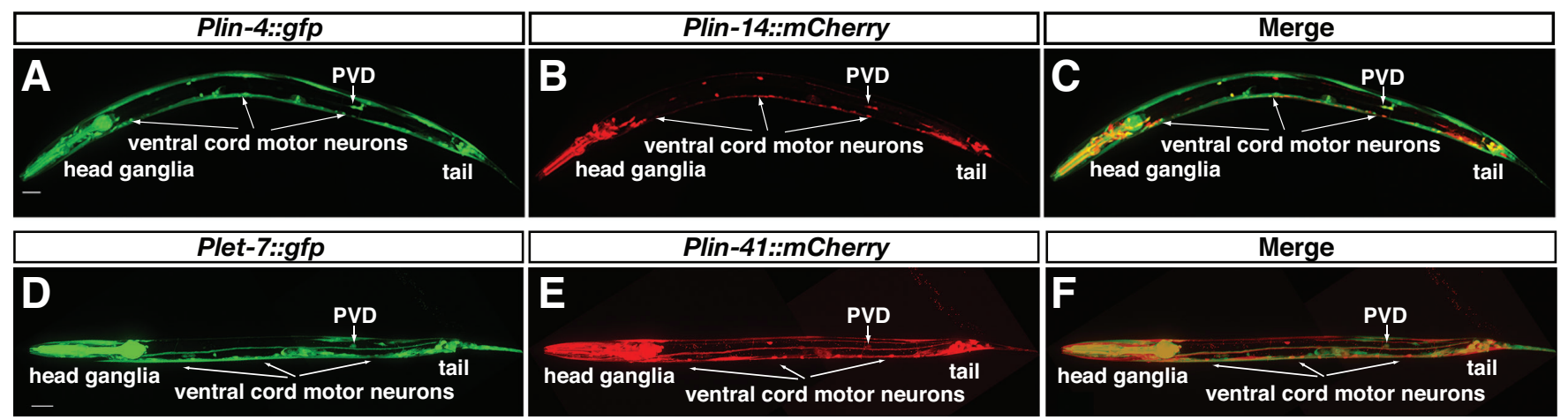

\section{G}

\section{L2 late L3 early mid late L4 early}

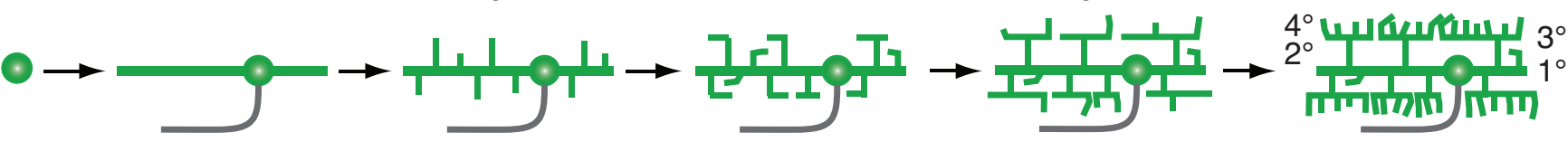

H
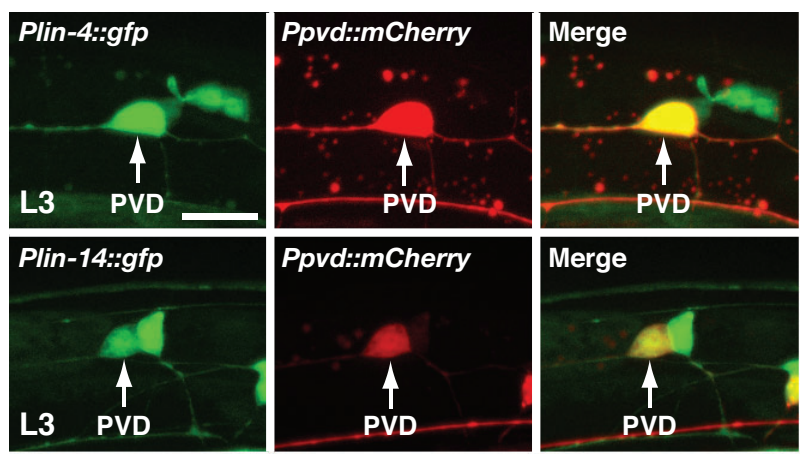

$\mathbf{J}$
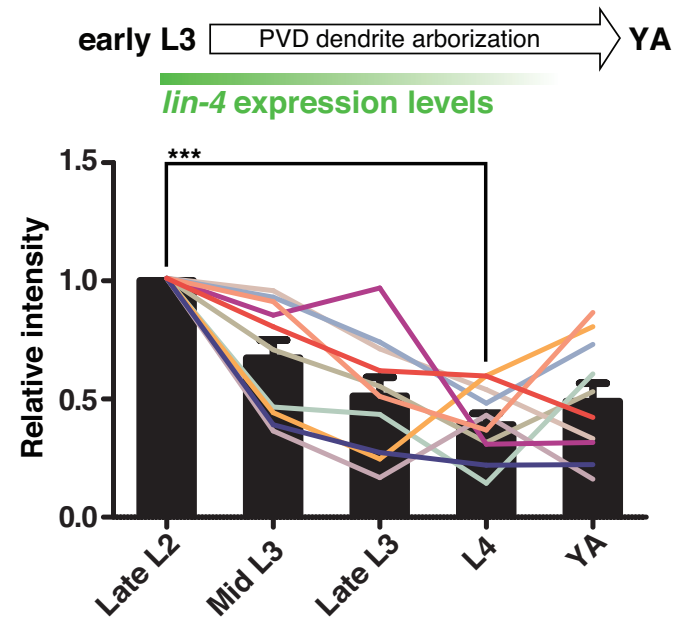

I

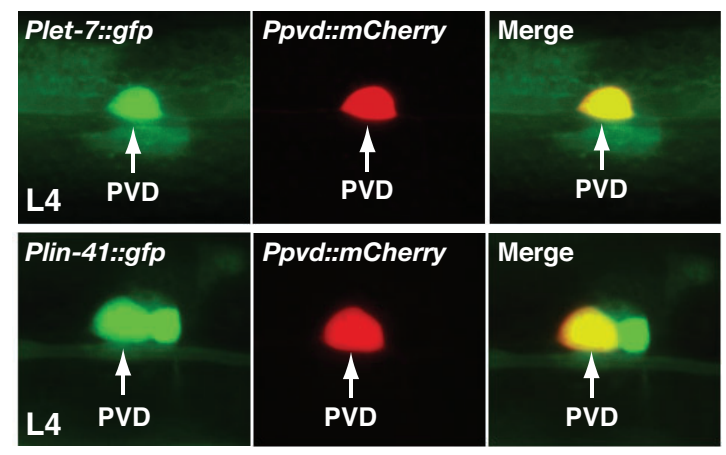



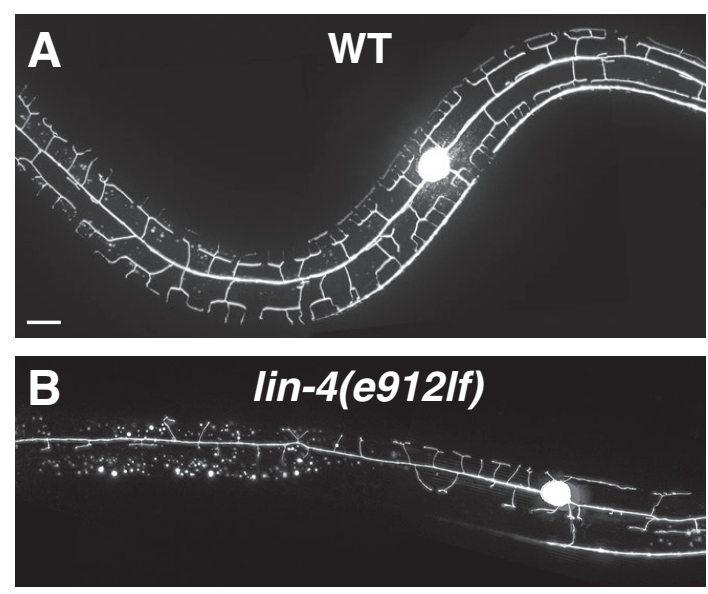

E

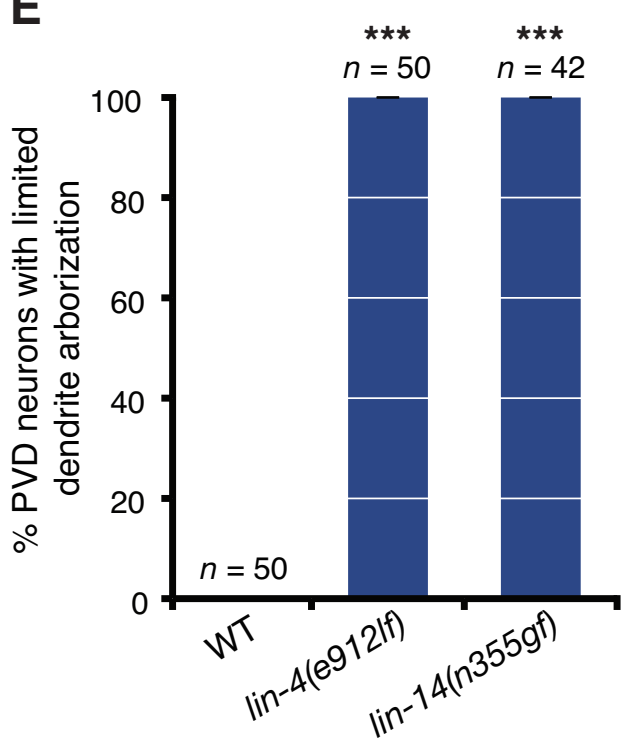

$\mathbf{F}$
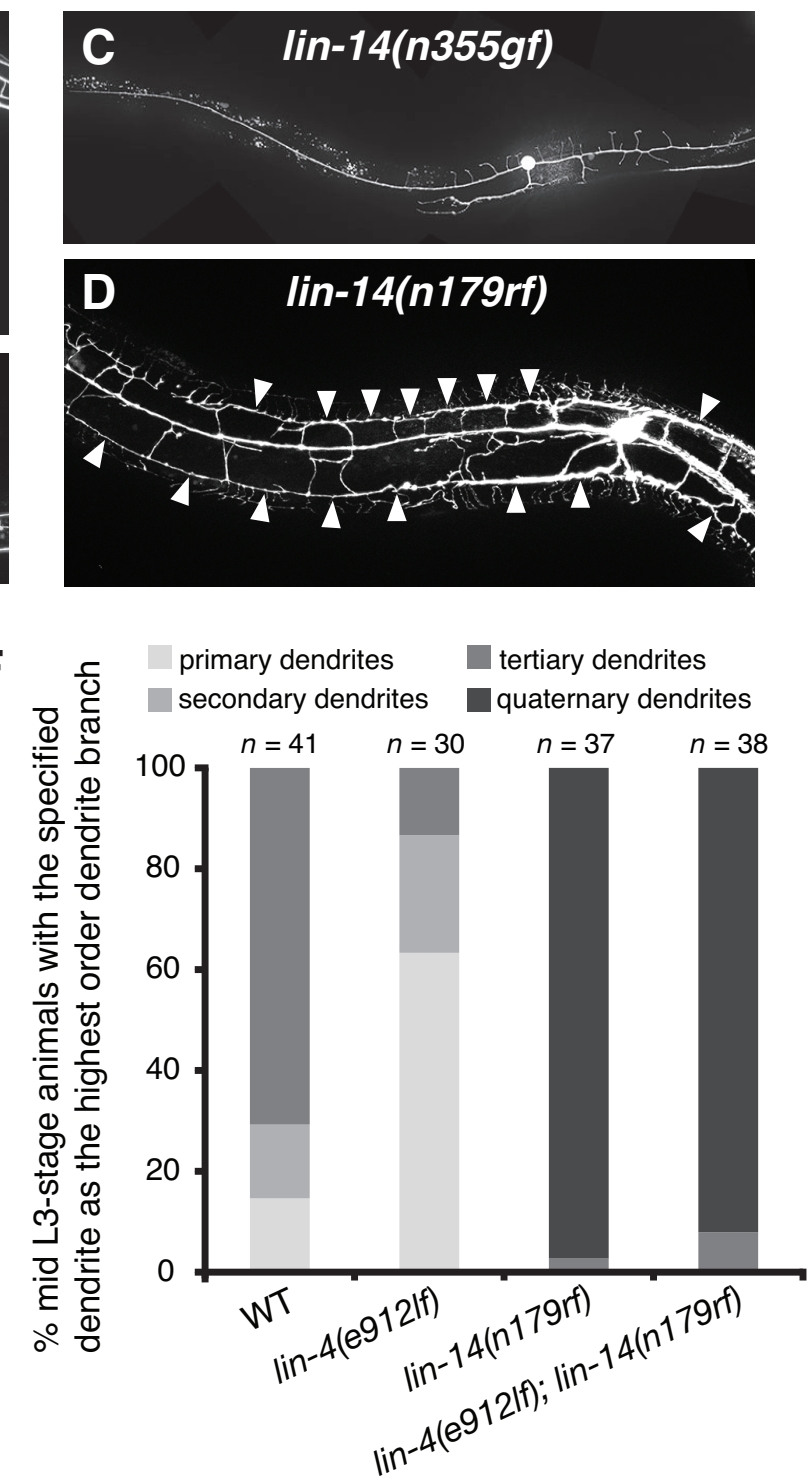

G Overlapped tertiary

non-overlapped tertiary

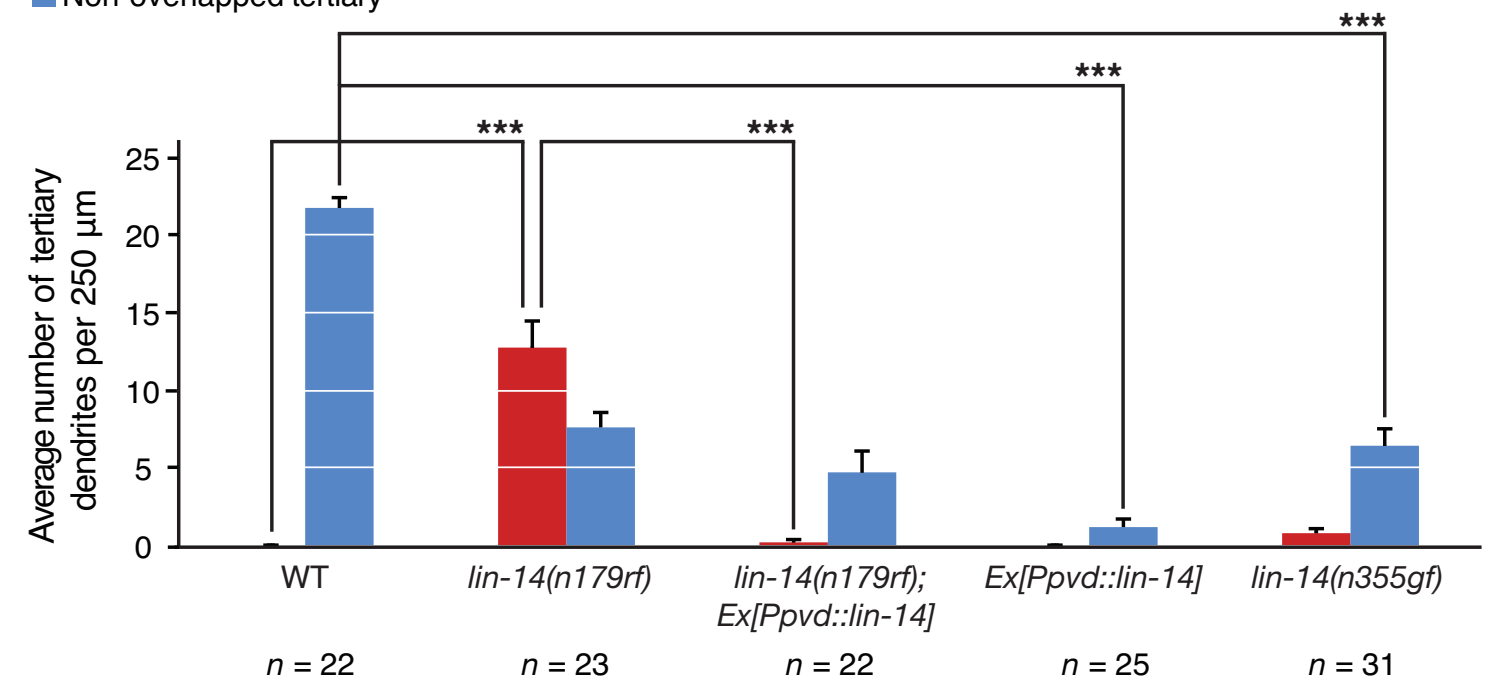

Figure 2 (Chang) 

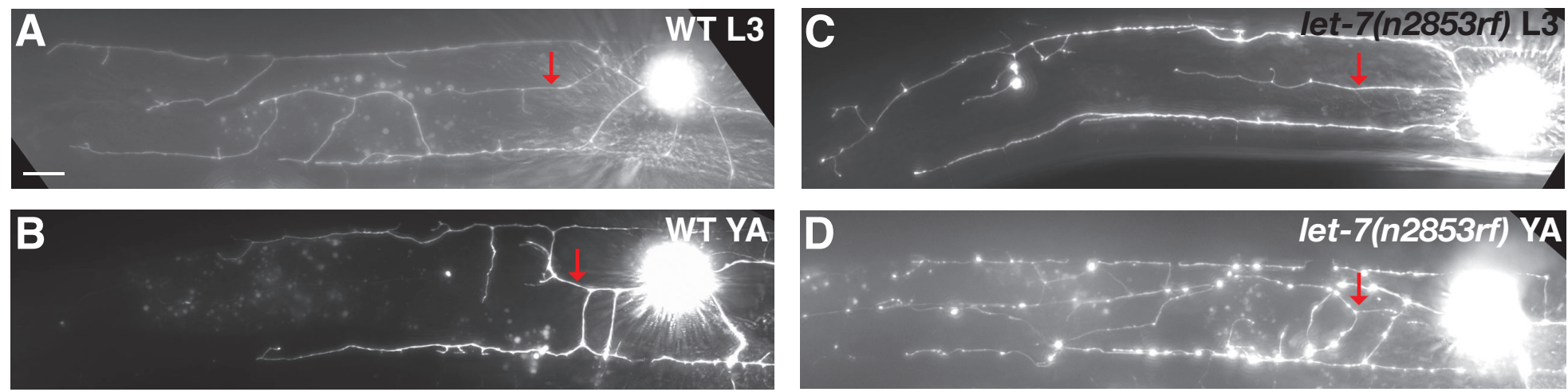

E

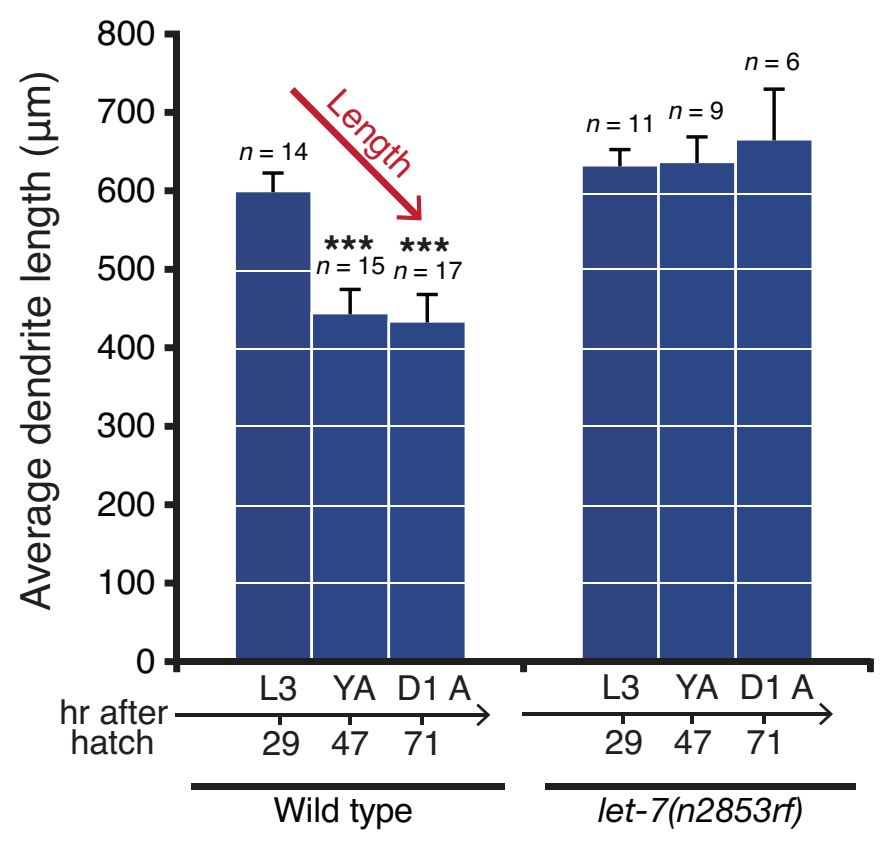

G

lin-28fosmid::GFP

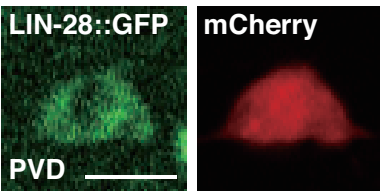

H
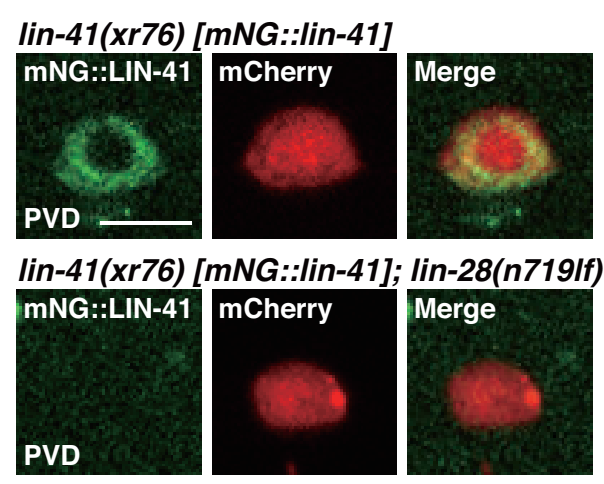

$\mathbf{F}$

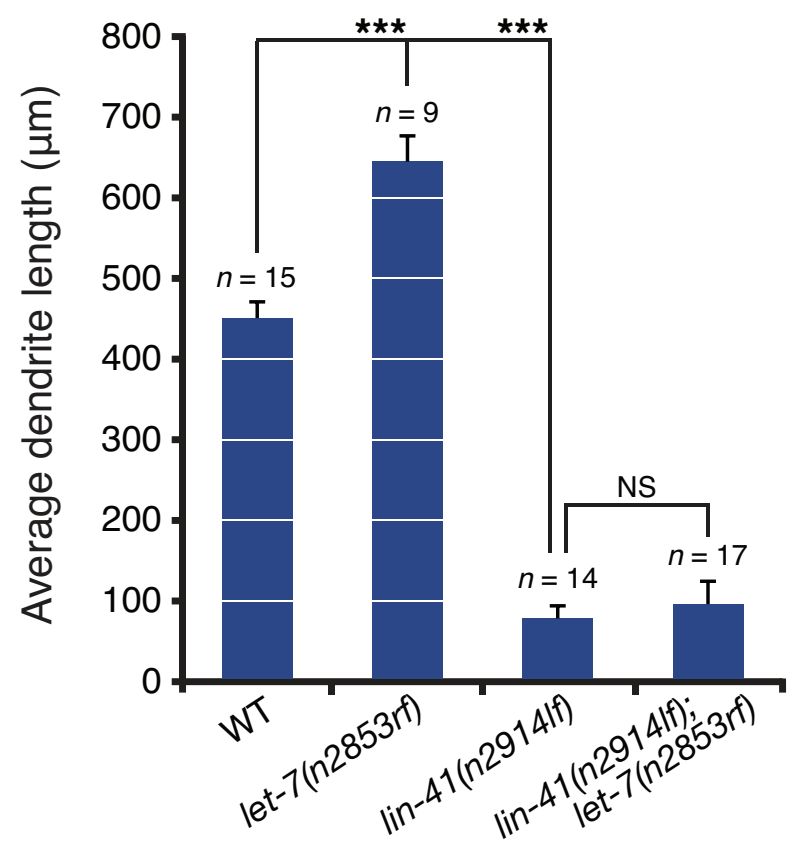

$\mathbf{J}$

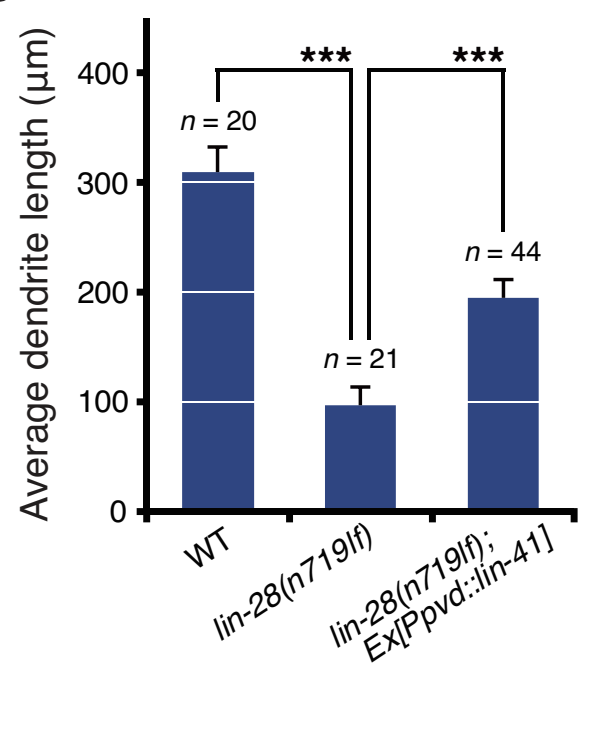


bioRxiv preprint doi: https://doi.org/10.1101/2021.08.31.458402; this version posted September 1,2021 . The copyright holder for this preprint (which was not certified by peer review) is the author/funder, who has granted bioRxiv a license to display the preprint in perpetuity. It is made available under aCC-BY-NC 4.0 International license.

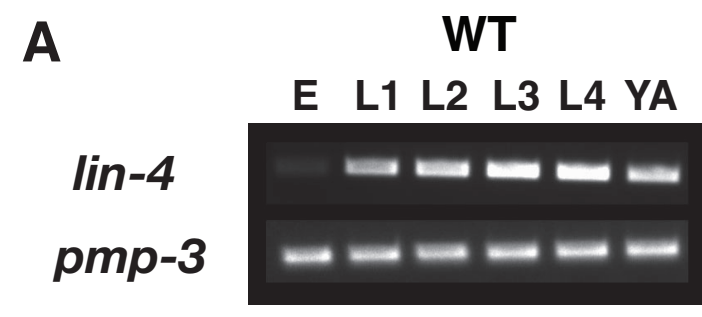

lin-4(xr71)
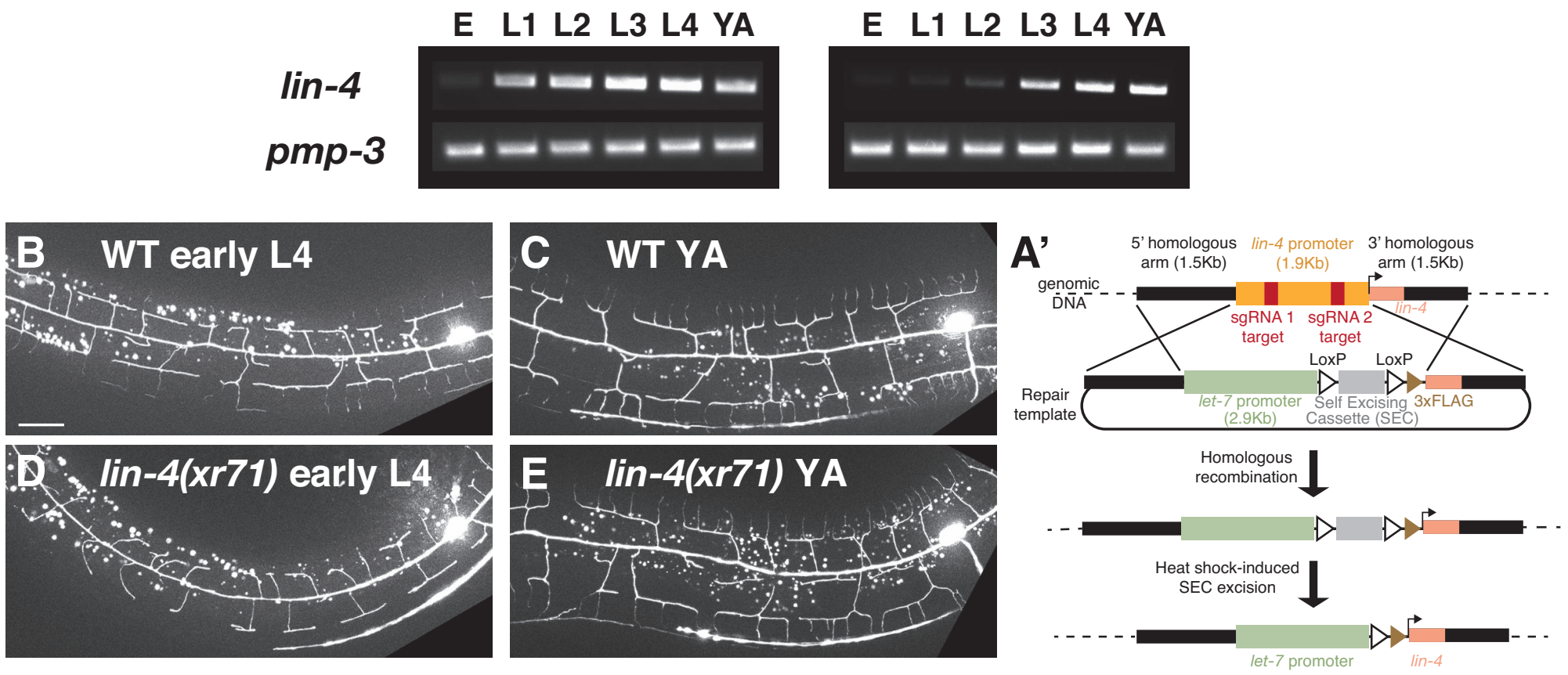

F

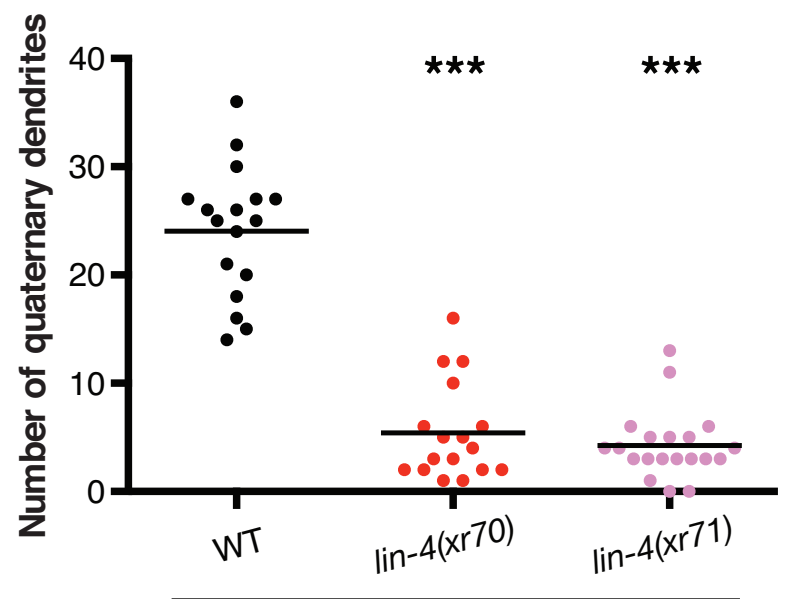

Early L4

H

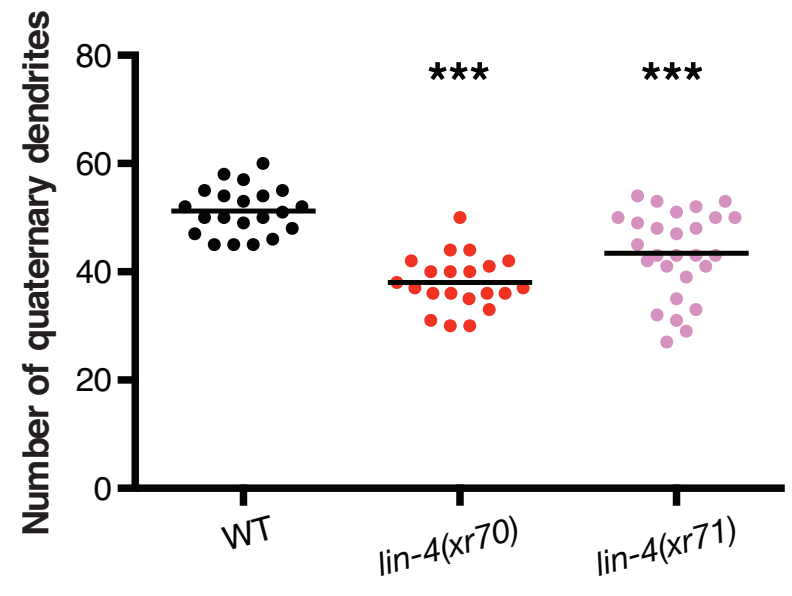

Adult 5hrs
G

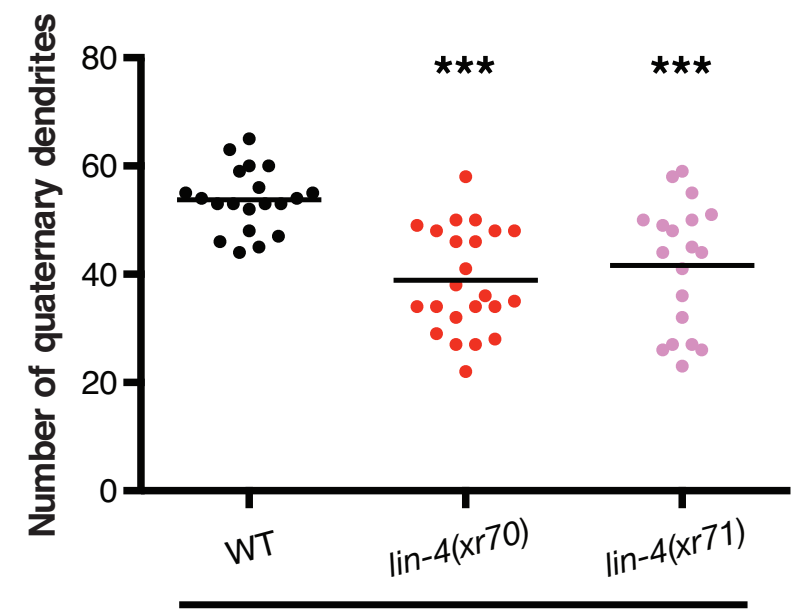

Adult Ohr

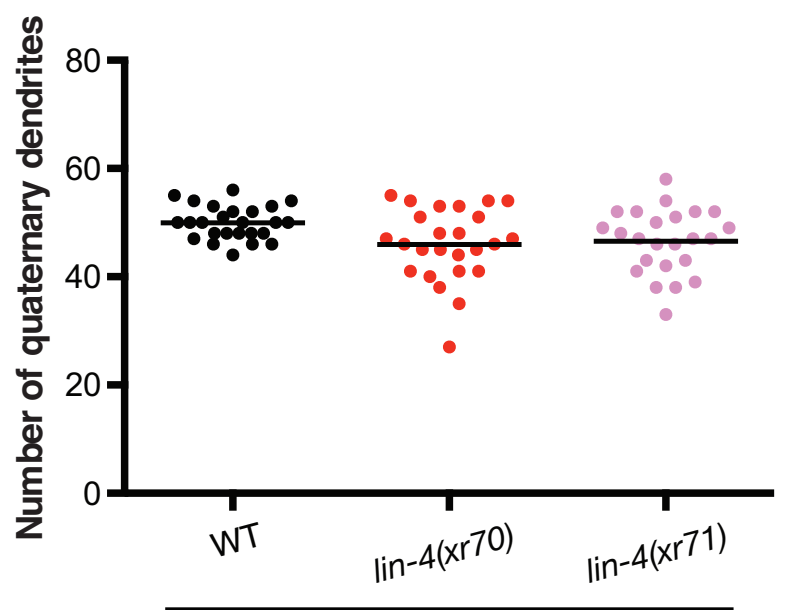

Adult 10hrs

Figure 4 (Chang) 
bioRxiv preprint doi: https://doi.org/10.1101/2021.08.31.458402; this version posted September 1, 2021. The copyright holder for this preprint (which was not certified by peer review) is the author/funder, who has granted bioRxiv a license to display the preprint in perpetuity. It is made available under aCC-BY-NC 4.0 International license.

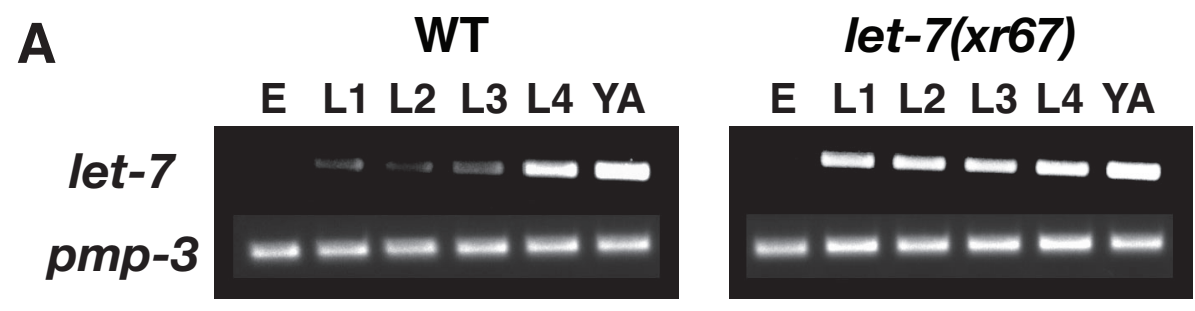

B

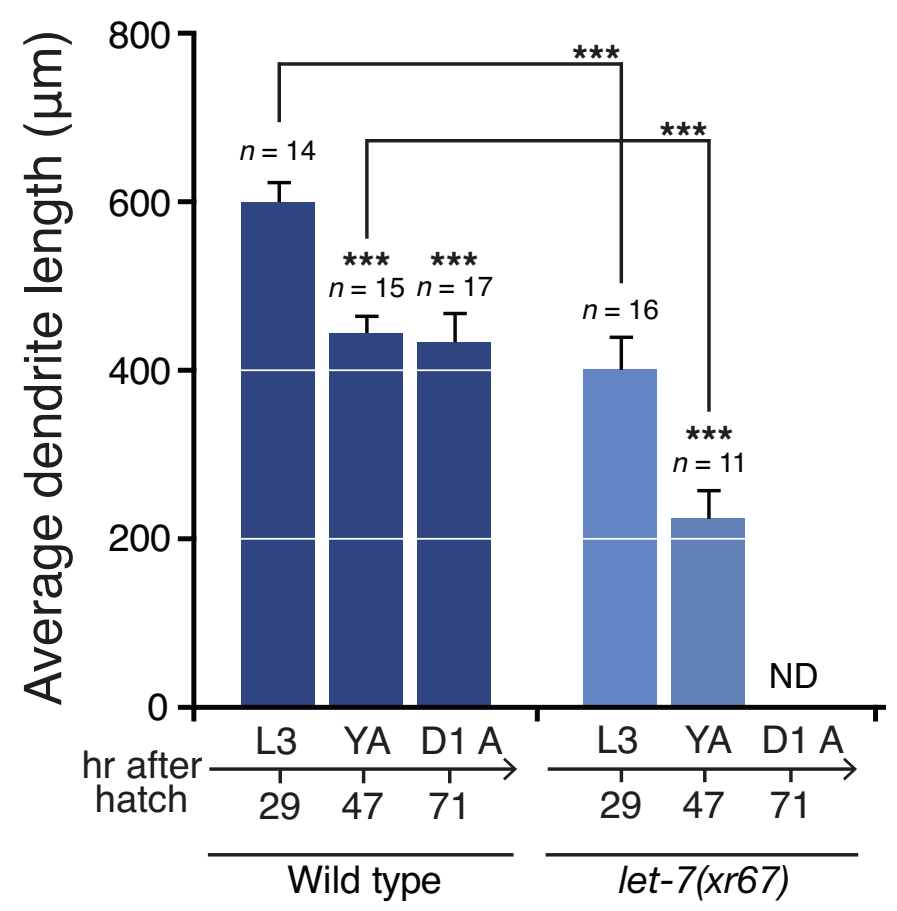

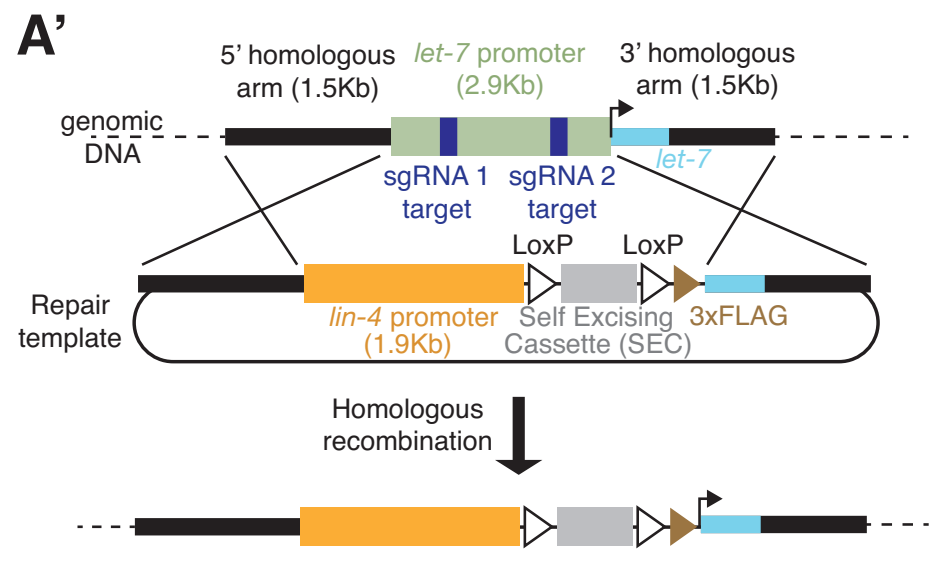

Heat shock-induced SEC excision 

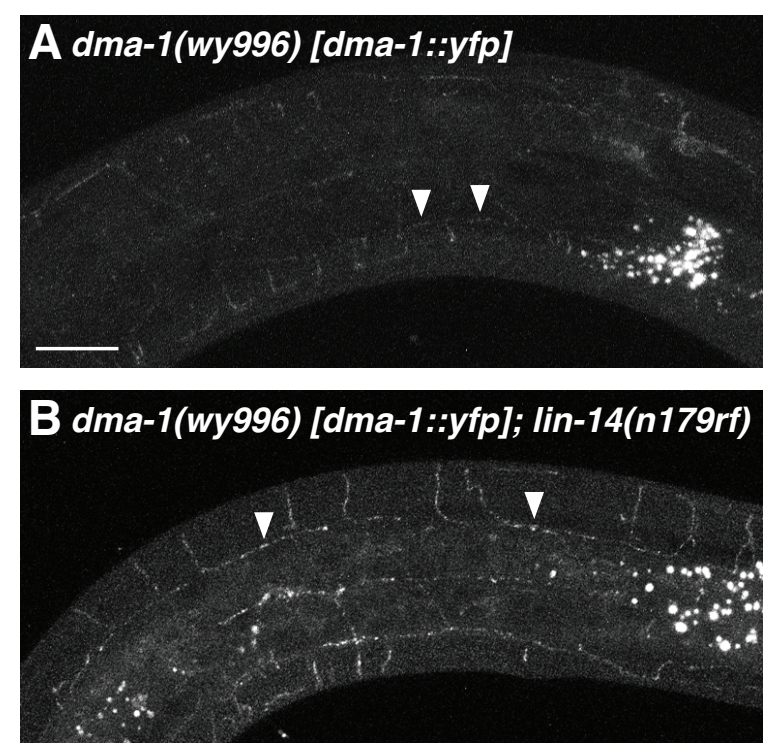

D

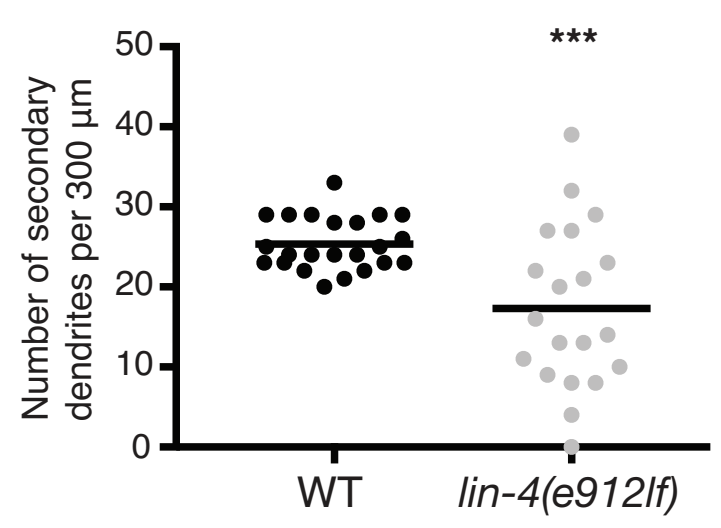

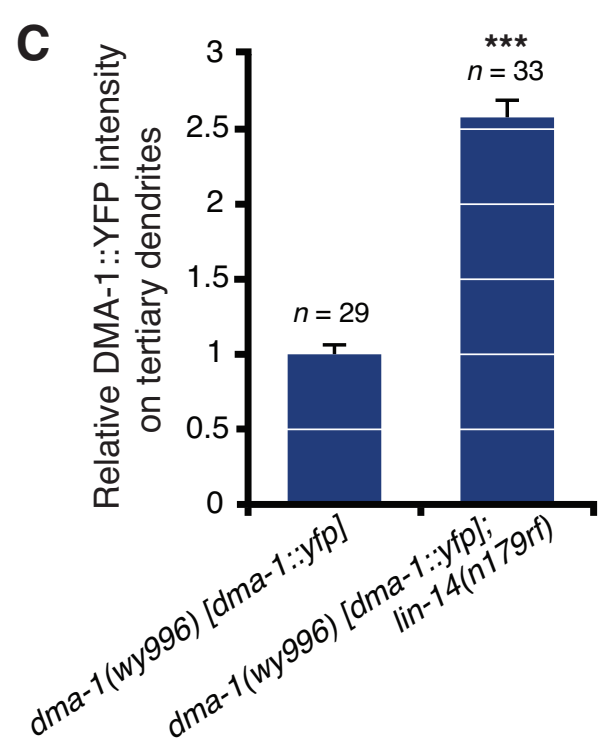

E $\quad \begin{aligned} & \text { excessive } \\ & \text { dendrites }\end{aligned} \quad \begin{aligned} & \text { limited } \\ & \text { dendrites }\end{aligned}$

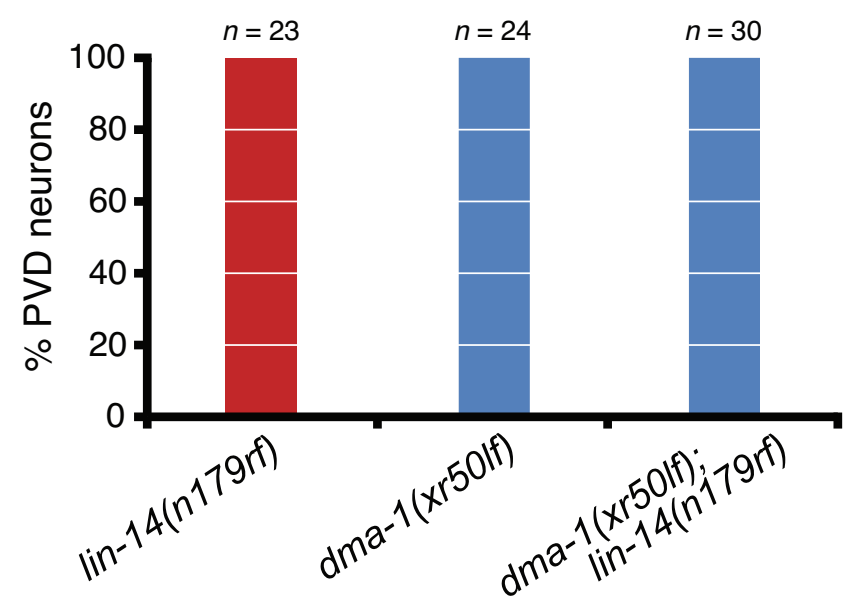




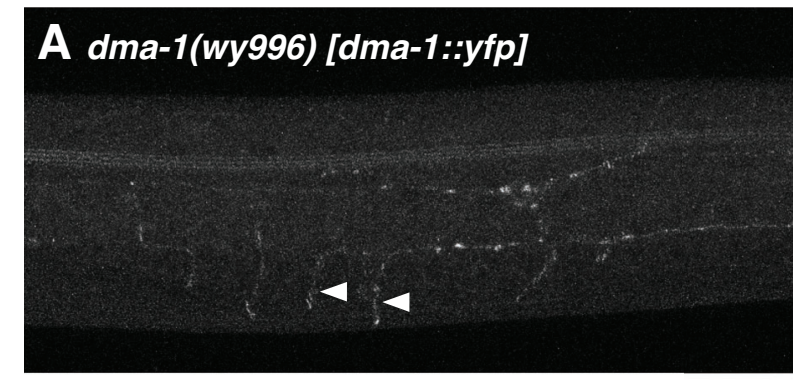

\section{B dma-1(wy996) [dma-1::yfp] lin-41(n2914lf)}

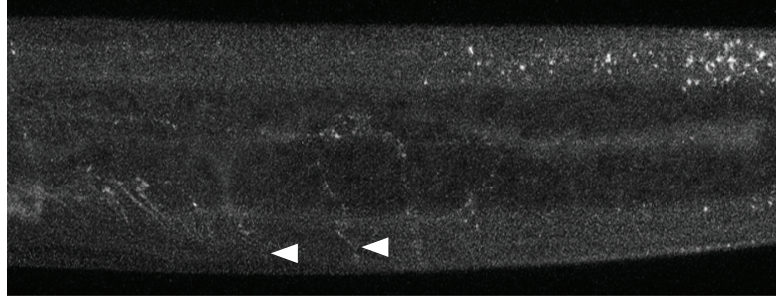

C

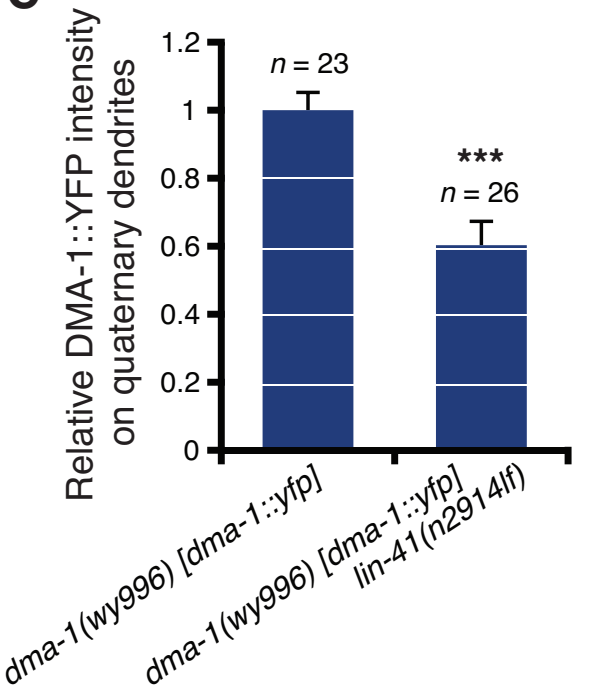

E

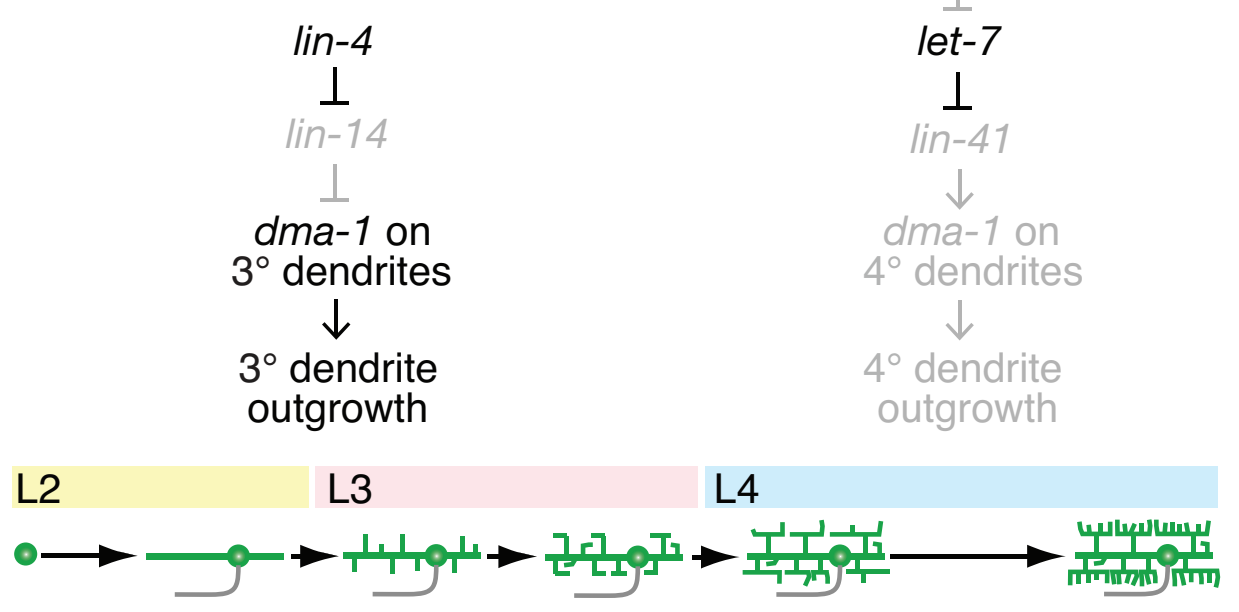

